\title{
INVENTARIO DE HYMENOPTERA (HEXAPODA) EN EL VENTORRILLO: UN RICO ENCLAVE DE BIODIVERSIDAD EN LA SIERRA DE GUADARRAMA (ESPAÑA CENTRAL)
}

\author{
J. L. Nieves-Aldrey*, F. Fontal-Cazalla, A. M. Garrido-Torres y C. Rey del Castillo
}

\begin{abstract}
RESUMEN
Se presentan los resultados globales del inventario de Hymenoptera llevado a cabo en el área de la Estación Biogeológica de El Ventorrillo, un área natural enclavada a 1450 metros de altitud en el sector central madrileño de la Sierra de Guadarrama. Con un programa de muestreo basado fundamentalmente en el uso de trampas Malaise, y adicionalmente trampas bandeja coloreadas y redes de barrido, llevado a cabo entre los años 1988 y 1991, se colectaron más de 1.000 .000 de ejemplares de insectos que han sido ingresados en las colecciones del Museo Nacional de Ciencias Naturales de Madrid. Cerca de 600.000 ejemplares de insectos fueron separados hasta el nivel de orden, de los cuales 83.668 (un 13.8\%) correspondieron al orden Hymenoptera, siendo después de Diptera (450.000 ejemplares, un $77,5 \%$ del total) el orden de insectos más numeroso en los muestreos. En las muestras colectadas se han identificado hasta la fecha 49 familias 518 géneros y 1310 especies de Hymenoptera; la riqueza total de himenópteros en las muestras colectadas se estima en más de 2700 especies, y la del conjunto de insectos en el inventario del área estudiada se estima en unas 13.000 especies. La lista de especies identificadas de himenópteros, junto a la cifra del total de ejemplares colectado de cada una, se adjunta en un apéndice final del trabajo. Entre los resultados del inventario se han descrito 10 especies nuevas para la ciencia y otras se han identificadas como tales, si bien aún no han sido formalmente descritas; se han citado numerosas novedades para la fauna europea y peninsular, entre las que se cuentan al menos 33 géneros y más de 170 especies nuevas para la fauna de la Península Ibérica. La abundancia relativa de himenópteros en el área, medida por las capturas de trampas Malaise, demostró ser muy alta, con relación a los datos de la literatura, alcanzándose un máximo de 916 ejemplares por día en la trampa y periodo más productivos. Las familias más abundantes en el inventario resultaron ser, en orden decreciente de abundancia, Braconidae, Ichneumonidae, Mymaridae, Scelionidae, Apidae y Pteromalidae, representadas por un número de ejemplares que va de los cerca de 12.000, en el caso de Braconidae, a los casi 6000 individuos de la familia Pteromalidae. La familia con mayor número de especies entre las identificadas fue Pteromalidae, con 290, seguida de Ichneumonidae con 217 y Sphecidae y Eulophidae con 107 y 101 especies, respectivamente. Los resultados del inventario se comparan con los de otros similares llevados a cabo en España, Europa y América; se constatan los altos valores de abundancia y riqueza de himenópteros del inventario de El Ventorrillo con relación a los comparados. Los resultados del inventario, aún no completamente finalizado, ponen de manifiesto la extraordinaria diversidad entomológica del entorno de la Sierra de Guadarrama en el que se enclava la estación biológica, no obstante la reducida extensión del área muestreada, un habitat que probablemente es representativo de otros similares en el sector central de la Sierra de Guadarrama. Toda la zona está, sin embargo, bajo la potencial amenaza de impacto ambiental por la creciente presión humana y urbanística de la cercana urbe de Madrid. Se insta a los responsables de la conservación del Medio Ambiente en la Comunidad de Madrid a tomar medidas para garantizar la protección de este singular enclave de biodiversidad, y de su entorno, en la sierra madrileña. En este sentido, esperamos que este trabajo contribuya a apoyar la reciente propuesta de creación del Parque Nacional de la Sierra de Guadarrama.
\end{abstract}

Palabras clave: inventario, Hymenoptera, sierra de Guadarrama, España central, biodiversidad, trampas Malaise.

\footnotetext{
* Museo Nacional de Ciencias Naturales (C.S.I.C). J. Gutiérrez Abascal 2, 28006 Madrid. e-mail: aldrey@mncn.csic.es
} 


\section{ABSTRACT \\ Inventory of Hymenoptera (Hexapoda) from "El Ventorrillo": a rich biodiversity spot in the Sierra de Guadarrama (central Spain)}

Overall data of the inventory of Hymenoptera from the Biogeological Station of "El Ventorrillo" are presented. The studied site is located at an altitude of $1450 \mathrm{~m}$, on the south face of the Sierra de Guadarrama (Central Spain), about 60 km NW from Madrid. Between 1988 and 1991 an insect biodiversity inventory was carried out using three sampling methods: Malaise traps, yellow pan traps and sweep nets. Out of the more than 1,000,000 insects trapped, increasing the collections of the MNCN, about 600,000 were sorted to order. We found 83,688 individuals of Hymenoptera (representing 13,8\% and the second more abundant group in the samples, after Diptera (450,000 individuals and 77,5\% of total). Forty nine families, 518 genera and 1310 species de Hymenoptera has been identified until now. The overall richness of Hymenoptera from El Ventorrillo is estimated in 2700 species and about 13,000 the number of insect species from the study site. An appendix is provided with the list of identified species and its overall abundance in the samples. As results of the inventory, ten new species for science have been described, and several more new species are not yet described; additionally, at least 33 genera and more than 170 species were recorded for the first time for Iberia. The abundance of Hymenoptera, as measured by Malaise trap catches, was very high, comparatively to other published data, reaching a peak of 916 individuals per trap day at the most productive trap and sampling period. The more abundant families were, in decreasing order, Braconidae, Ichneumonidae, Mymaridae, Scelionidae, Apidae and Pteromalidae, represented by numbers of individuals between 12,000, for Braconidae, to near 6000 for Pteromalidae. Among the identified families, the more species rich at the study area were in decreasing order: Pteromalidae (290), Ichneumonidae (217), Sphecidae (107) and Eulophidae (101 species). The richness of the 29 remaining families at the area of study was estimated extrapolating overall regional richness data from Spain. The hymenopteran numbers from the inventory are compared with other local richness data from Spain and other surveys from Europe and America. The comparatively high abundance and richness figures at El Ventorrillo are emphasized. Results of the inventory, not yet completed, indicate a very high entomological diversity at the surveyed site, a small relatively well preserved area less than $0.5 \mathrm{Km}^{2}$, that probably is representative of other similar habitats in the Sierra of Guadarrama. This area is, however, under potential menace of alteration by the growing human and urban pressure of the vicinity of Madrid. We urge the politics and nature managers to protect this rich biodiversity spot. We hope that this work can help to support the recent proposal of creation of the National Park of the Sierra of Guadarrama.

Key words: inventory, Hymenoptera, sierra de Guadarrama, central Spain, biodiversity, malaise traps.

\section{Introducción}

Una de las facetas más fascinantes de la Vida sobre la tierra es su asombrosa diversidad. Concepto multidimensional, la Biodiversidad, o variedad de la vida, se expresa en distintos niveles de organización, desde genes a ecosistemas (Purvis \& Hector, 2000); sin embargo, la medida más usual de la biodiversidad es la riqueza o número de especies de una localidad, habitat, ecosistema o región (Gaston, 1996, 2000). Por paradójico que pueda parecer en los albores del tercer milenio y tras más de trescientos años de dar nombre y describir las especies vivientes, es un hecho constatado que sólo una pequeña fracción de las especies que pueblan la biosfera han sido descritas. Esta diversidad desconocida estaría formada esencialmente por artrópodos, y más concretamente por insectos, el grupo de organismos que, con cerca de un millón de especies conocidas, es con gran diferencia el componente mayoritario del conjunto de especies que pueblan el planeta.

Por otra parte, la denominada "crisis de la biodiversidad" debida a la rápida y creciente degradación de las áreas naturales y la subsiguiente pérdida de diversidad biológica que se está produciendo, especialmente en los ecosistemas tropicales, ha provocado un redoblado interés entre la comunidad científica por acelerar el estudio y descripción de la biodiversidad en todas sus facetas (Gaston, 1996; Tilman, 2000; Purvis \& Hector, 2000). Dentro de este marco, la realización de inventarios de biodiversidad, a distintos niveles, es una de las tareas básicas, cuya importancia ha sido resaltada por muchos autores (May, 1988; Yoon, 1993; Stork et al., 1996). Los datos de los inventarios de flora y 
fauna son la base más firme para tomar decisiones fundamentadas en cuanto a la conservación de los habitat y, por otra parte, se ha subrayado que la extrapolación de datos de inventarios locales de taxones puede ser en última instancia un medio de estimación de la riqueza global de especies (Colwell \& Coddington, 1994; Stork et al., 1996; Godfray et al., 1999). Gran parte del esfuerzo de inventario actual se está realizando en áreas tropicales, con mucho las más desconocidas. Sin embargo, aunque las áreas templadas del globo se conocen mucho mejor en lo que concierne a sus componentes de especies y, no obstante la larga tradición de investigación entomológica en los países templados, los inventarios generales locales de insectos son escasos o inexistentes. También son escasos los datos globales, sistemáticamente colectados de abundancia, riqueza y distribución para la mayoría de los grandes grupos de insectos. La razón fundamental de este hecho es la extraordinaria diversidad de los insectos, que a su vez condiciona una especialización extrema de los entomólogos. Sin embargo el paulatino declive del trabajo taxonómico, registrado desde hace décadas, ha conllevado una notoria escasez de taxónomos especialistas, los únicos que pueden garantizar la identificación de grandes grupos de organismos deficientemente conocidos, especialmente de insectos. Este hecho ha sido repetidamente advertido como uno de los principales obstáculos para acometer con garantías de éxito el inventario global de la Biodiversidad subrayando la acuciante necesidad de programas de formación de taxónomos en grupos hiperdiversos de organismos (Holden, 1989; Stork et al., 1996). Por otra parte, el trabajo de cualquier inventario completo local de insectos conlleva un esfuerzo ingente en recursos y tiempo, y es muy difícil reunir el esfuerzo humano y material necesario para acometer la empresa con garantías de éxito.

La dificultad de realizar inventarios completos de biodiversidad al nivel local hace que las decisiones sobre conservación se tomen con datos de organismos emblemáticos, bien conocidos, como vertebrados o plantas vasculares ignorando el papel de organismos como los insectos, que forman el principal componente de la biodiversidad de cualquier hábitat terrestre.

Hymenoptera es uno de los cuatro órdenes hiperdiversos de insectos, El número de especies descritas sobrepasa las 120.000 rivalizando con Coleoptera por el puesto del orden de insectos más rico en especies (Gaston, 1993; Gauld \& Hanson, 1995). Se cree, sin embargo, que queda por descubrir una fracción muy importante de la diversidad de este grupo de insectos, especialmente en los trópicos y de las familias parasitoides, y se estima que su riqueza real sobrepasaría el medio millón de especies (Gaston et al., 1996). Los inventarios completos a nivel local o regional de Hymenoptera son muy escasos. Aparte de algunos de zonas tropicales, como Sulawesi (Noyes, 1989a, 1989b) y Costa Rica (Gaston et al., 1996), existen pocos inventarios en las regiones templadas. Entre los ejemplos relativamente recientes se pueden resaltar el llevado a cabo en una zona pantanosa en Alberta (Canadá) (Finnamore, 1994) y el del Parque Nacional Yellowstone (Lockwood et al., 1996) en América del Norte. En Europa se han publicado varios inventarios realizados en Holanda (Zuiljen, 1996) y Alemania (Ulrich, 1999). Por lo que se refiere a España el único inventario general de Hymenoptera de un área geográfica publicado hasta la fecha es el realizado en un sabinar de Retuerta de Pina en la región de los Monegros (Zaragoza) (Melic \% Blasco-Zumeta, eds., 1999).

En los últimos años hemos desarrollado proyectos de investigación centrados en el inventario de Insecta, particularmente Hymenoptera, en áreas templadas de España y tropicales de Panamá. Presentamos aquí los resultados del inventario realizado en la Estación Biogeológica de El Ventorrillo (Madrid), en el sector central de la Sierra de Guadarrama. El proyecto, financiado por la Comunidad de Madrid e iniciado en 1991, tenía como objetivo principal el inventario, tan completo como fuera posible, de la entomofauna de un enclave natural emblemático de la Comunidad de Madrid: el sector central de la Sierra de Guadarrama en la vertiente sur, o madrileña. Se esperaba conseguir con los datos del inventario los siguientes objetivos complementarios: 1) comparación con inventarios similares de otras localidades o hábitat; 2) Obtener cifras fiables para extrapolación de los resultados para la estimación de riqueza de insectos a nivel regional y del conjunto de Iberia; 3) Obtener colecciones de base para un seguimiento y control futuro de cambios en la biodiversidad del área estudiada, por posible impacto humano o cambio climático, por el fenómeno conocido como "calentamiento global". Por razón de la composición del equipo nos hemos concentrado en el inventario del orden Hymenoptera. Algunos resultados parciales del inventario fueron ya publicados en años precedentes (Nieves-Aldrey \& Rey del Castillo, 1991; Nieves-Aldrey, 1995; Sanchís et al., 1995; Pujade-Villar et al., 1998; Garrido Torres \& Nieves-Aldrey, 1992, 1999; Buhl \& Nieves-Aldrey, 2001). Ahora se presentan los resultados globales del inventario de este orden de insectos. 


\section{ÁREA DE ESTUDIO}

Los muestreos fueron efectuados desde 1988 a 1991 en la Estación Biogeológica de El Ventorrillo dependiente del Museo Nacional de Ciencias Naturales de Madrid (CSIC). La estación es un recinto acotado de aproximadamente seis hectáreas de superficie, enclavado en la vertiente sur de la Sierra de Guadarrama, en el término municipal de Cercedilla (UTM 30TVL1412), a 1450 metros de altitud, en la carretera de acceso al puerto de Navacerrada, a unos 60 kilómetros al noroeste de Madrid. La estación se sitúa en una ladera orientada al suroeste por encima de unos pastizales que forman un pequeña vaguada. Desde el punto de vista fitoclimático el lugar se sitúa dentro de la serie supramediterránea cántabro-ibérico-ayllonense húmeda silicícola del roble melojo (Festuco heterophyllaeQuercetum pyrenaicae S.) (Rivas Martínez, 1982). Sin embargo la vegetación potencial de este piso ha sido alterada y está ocupada actualmente en gran parte, por repoblación antigua de Pinus sylvestris L. En el recinto de la estación, existe una densa vegetación arbórea, en su mayor parte de origen artificial, integrada fundamentalmente por arces (Acer pseudoplatanus) y olmos de montaña, encontrándose también tilos, pinos, cedros, abetos, chopos, fresnos y acebos. Esta densa mancha de vegetación, se interrumpe bruscamente en el límite inferior del recinto de la estación, dando paso a unos pastizales con rosaledas y zarzales. La zona de transición está ocupada por una variada vegetación arbustiva con especies de Cytisus, Crataegus, Rubus, Rosa, Cistus, Santolina, Juniperus, Prunus etc y, ya fuera de los terrenos de la estación, aparecen algunos manchas de Quercus pyrenaica con jarales. El clima de la zona es de tipo mediterráneo subhúmedo de tendencia centroeuropea (Allué Andrade, 1987). Los datos de la estación meteorológica más cercana, correspondiente al Puerto de Navacerrada (1890 m.), la ubican dentro del piso bioclimático supramediterráneo, caracterizado por un invierno de tipo frío, con posibles heladas de septiembre a junio y ombroclima húmedo (con precipitación de 1.000 a $1.600 \mathrm{~mm}$. al año) con un corto período de sequía estival en julio-agosto coincidiendo con el período más cálido y libre de heladas. Dada su proximidad geográfica, en líneas generales estos datos no deben diferir sustancialmente de los registrados en la zona de estudio, si bien cabe esperar, debido a la diferencia de altitud, un incremento de las temperaturas, así como una reducción del índice de pluviosidad en el área de El Ventorrillo.

La estación de El Ventorrillo está situada en el corazón de la Sierra de Guadarrama, una de las áreas geográficas de mayor biodiversidad dentro de la
Península Ibérica, como ha sido registrado, por ejemplo, para plantas vasculares (Lobo et al., 2001) y grupos de insectos, como los escarabajos coprófagos (Lobo \& Martín-Piera, 2002). Las altas cifras de especiación y endemicidad de insectos de esta área geográfica se han puesto también de manifiesto por distintos autores (Martín-Cano \& Gurrea, 1995; García-Barros et al., 2002). A una escala geográfica más amplia, se ha señalado que la Península Ibérica es una de las regiones de Europa que albergan una más rica diversidad animal (Ramos et al., 2001), región a su vez que forma parte de la Cuenca Mediterránea, uno de los más importantes "puntos calientes" de Biodiversidad del Globo (Balleto \& Casale, 1991; Myers \& Cowling, 1999).

\section{Materiales y métodos}

Los muestreos se extendieron desde mayo de 1988 a Julio de 1991. La relación de métodos y periodos de muestreos, tipos de trampas y ubicaciones se relacionan en la tabla I. La mayor parte del material fue colectado por medio de trampas Malaise de origen comercial, de luz de malla fina y coloración completamente negra (Townes, 1972; Darling \& Parker, 1988). Complementariamente se utilizaron trampas bandeja coloreadas de amarillo y en un periodo de muestreo intensivo de una semana de duración en julio de 1991 se utilizaron redes entomológicas de barrido de diseño de Boucek (Noyes, 1982). Una de las trampas Malaise se mantuvo operando de manera continua un ciclo anual completo, con excepción de los meses invernales de diciembre a febrero. Los frascos colectores de las trampas Malaise conteniendo las muestras en alcohol al 75\% eran reemplazados semanalmente; El material estudiado está depositados en las colecciones del Museo Nacional de Ciencias Naturales, en Madrid (MNCN) con excepción de pequeñas representaciones de los ejemplares que fueron retenidas en las colecciones personales o institucionales de los investigadores que identificaron las distintas familias de himenópteros.

\section{Resultados}

\section{ABUNDANCIA}

En el programa de muestreo con trampas Malaise el número de insectos colectados, separados e identificados hasta el nivel de orden fue de 581.271. Por lo que respecta al orden Hymenoptera el total de individuos colectado fue de 83.668, un 
Tabla I.- Resumen de datos de muestreo: ubicaciones, tipos de trampas y métodos empleados, fechas y días de muestreos. Las abreviaturas corresponden a modelos de trampas Malaise: TMB, trampa Malaise blanca; TMNA, negra, modelo americano; TMNI, negra modelo inglés.

Table I.- Summary of sampling data: sites, traps, sampling methods and sampling periods. Abbreviations corresponds to Malaise traps models, TMB, malaise trap all white, TMNI, malaise trap black, commercial origin USA, TMNI, malaise trap all black, commercial origin GB.

\begin{tabular}{|c|c|c|c|c|c|c|c|}
\hline Localidad & Hábitat & Ubicación & Método & $\begin{array}{l}\text { Número } \\
\text { de trampas }\end{array}$ & $\begin{array}{c}\text { Coloración } \\
\text { y malla }\end{array}$ & $\begin{array}{c}\text { Período } \\
\text { de muestreo }\end{array}$ & Días \\
\hline El Ventorrillo & $\begin{array}{l}\text { Linde pradera- } \\
\text { Bosque mixto }\end{array}$ & 1 (más xérica) & TMB & 1 & Blanca, fina & 20/V-6/X, 1988 & 137 \\
\hline El Ventorrillo & “ & 2 (más húmeda) & TMNA & 1 & Violácea, fina & VI, 1989-VI, 1990 & 240 \\
\hline El Ventorrillo & “ & Todo el recinto & Trampas bandeja & 5 & Amarilla & 16/IV-16/VI, 1990 & 260 \\
\hline El Ventorrillo & “ & 1 & TMNI & 1 & Negra & 9-17/VII, 1991 & 8 \\
\hline El Ventorrillo & “ & 2 & TMNI & 1 & Negra & 9-17/VII, 1991 & 8 \\
\hline El Ventorrillo & “ & Todo el recinto & Trampas bandeja & 4 & Amarilla & 9-17/VII, 1991 & 32 \\
\hline El Ventorrillo & “ & Todo el recinto & Barrido & & & 9-17/VII, 1991 & \\
\hline
\end{tabular}

$13.8 \%$ del total, siendo después de Diptera $(450.000$ ejemplares, el $77,5 \%$ ) el orden de insectos más numeroso en las muestras de trampa Malaise. Más ilustrativas de la abundancia de Insecta e Hymenoptera en el área estudiada son las cifras de eficiencia de captura de las trampas Malaise, medidas en número de ejemplares colectados por día de muestreo. En el periodo más productivo, la primera quincena de julio, una sola trampa Malaise capturó 9988 ejemplares de insectos en un día y, por lo que respecta a himenópteros, el record es una cifra de 915 ejemplares por trampa y día, el día 10 de julio en el emplazamiento 1. La media de ejemplares capturados por día de trampa Malaise en el periodo de 9 de junio a 11 de noviembre de 1989 (155 días de trampa) fue de 2617 ejemplares de insectos. El total de insectos capturado por dicha trampa Malaise, en el emplazamiento 2 a lo largo de un año (junio de 1989 a junio de 1990) fue de 554.632 ejemplares, de los cuales 61.720 correspondieron a Hymenoptera. Estas cifras superan notablemente los datos publicados de muestreos similares efectuados en otros lugares y países, tanto de latitudes templadas como tropicales (Owen, 1983; Hammond, 1990; Mathews \& Mathews, 1970; Pujade-Villar, 1996; Segade et al., 1997; Tomé et al., 2001). Para cifras comparativas véase la tabla VI en Nieves-Aldrey \& Fontal-Cazalla (1997).

\section{RiQUEZA Y DIVERSIDAD DE HYMENOPTERA}

Del total de 61 familias de Hymenoptera representadas en España, 49 están presentes en las muestras colectadas en el área inventariada. Hasta la fecha hemos identificado 1310 especies corres- pondientes a 38 de las 49 familias de himenópteros colectadas en el área representando a todas las superfamilias del grupo.

En la tabla II figura la relación de familias de Hymenoptera inventariadas en el área estudiada. Para cada familia se dan las cifras totales respectivas de abundancia (número de ejemplares) y riqueza (número de especies) en el conjunto de las muestras. Las familias más abundantes resultaron ser las dos de la superfamilia Ichneumonoidea: Braconidae e Ichneumonidae, con cerca de 12.000 y 10.600 ejemplares colectados, respectivamente. Casi con el mismo número de individuos que Ichneumonidae figura a continuación la familia Mymaridae (Chalcidoidea) seguida a corta distancia de la familia Scelionidae (Proctotrupoidea). Los Apidae (Apoidea) y otras dos familias de calcidoideos: Pteromalidae y Eulophidae se situan en $6^{\circ}$ y $7^{\circ}$ lugar en orden de abundancia, seguidas de Platygastridae y Tenthredinidae ambas superando los 2000 ejemplares. Con más de 1000 individuos en las muestras figuran en orden decreciente Sphecidae, Trichogrammatidae, Figitidae, Encyrtidae y Ceraphronidae.

Al igual que se indica en la tabla II, el grado de identificación de Hymenoptera realizado hasta la fecha es desigual. 28 familias (en negrita en la tabla) han sido identificadas hasta el nivel de especie en el total de las muestras colectadas; por otra parte, 8 familias han sido identificadas hasta especie, ya sea en sólo una fracción de las muestras o abarcando solamente una subfamilia o grupo de géneros de dicha familia; 3 familias han sido identificadas total o parcialmente hasta el nivel de géne- 
Tabla II.- Relación de familias de Hymenoptera inventariadas en El Ventorrillo. La columna de la izquierda refleja el número de especies identificadas hasta el momento, la de la derecha el número de ejemplares colectado de cada familia en el total de los muestreos. Los números en negrita indican las familias que han sido identificadas en su totalidad.

Table II.- - List of families of Hymenoptera from the inventory of El Ventorrillo. Column on the left represent species number of families identified until now. Column on the right reflects number of individuals from totals of samples. Families that have been fully identified figure in bold.

\begin{tabular}{|c|c|c|c|c|c|}
\hline $\begin{array}{c}\text { Hymenoptera } \\
\text { Superfamilias } \\
\text { y Familias }\end{array}$ & $n^{\circ}$ de especies & $n^{0}$ de ejemp. & $\begin{array}{c}\text { Hymenoptera } \\
\text { Superfamilias } \\
\text { y Familias }\end{array}$ & $n^{0}$ de especies & $n^{0}$ de ejemp. \\
\hline XYELOIDEA & & & Encyrtidae & 19 & 1265 \\
\hline Xyelidae & 2 & 64 & Eulophidae & 101 & 5269 \\
\hline MEGALODONTOIDEA & & & Eupelmidae & 10 & 349 \\
\hline Megalodontidae & 2 & 2 & Eurytomidae & - & 287 \\
\hline TENTHREDINOIDEA & & & Leucospidae & 2 & 12 \\
\hline Argidae & 5 & 29 & Mymaridae & $2(\mathrm{~g})$ & 10608 \\
\hline Cimbicidae & 4 & 10 & Ormyridae & 4 & 39 \\
\hline Tenthredinidae & 98 & 2478 & Perilampidae & - & 12 \\
\hline CEPHOIDEA & & & Pteromalidae & 290 & 5892 \\
\hline Cephidae & 7 & 16 & Signiphoridae & 1 & 3 \\
\hline ORUSSOIDEA & & & Tetracampidae & 2 & 35 \\
\hline Orussidae & 1 & 1 & Torymidae & - & 515 \\
\hline EVANIOIDEA & & & Trichogrammatid & 1 & 1336 \\
\hline Evaniidae & 1 & 356 & ICHNEUMONOI & DEA & \\
\hline Gasteruptiidae & - & 85 & Braconidae & 26 & 11994 \\
\hline CERAPHRONOIDEA & & & Ichneumonidae & 217 & 10613 \\
\hline Ceraphronidae & - & 1195 & CHRYSIDOIDEA & & \\
\hline Megaspilidae & - & 1004 & Bethylidae & - & 128 \\
\hline PROCTOTRUPOIDEA & & & Chrysididae & 43 & 221 \\
\hline Heloridae & 1 & 15 & Dryinidae & 20 & 629 \\
\hline Diapriidae & - & 5171 & Embolemidae & 1 & 3 \\
\hline Proctotrupidae & - & 867 & VESPOIDEA & & \\
\hline PLATYGASTROIDEA & & & Formicidae & 28 & 807 \\
\hline Scelionidae & $14(\mathrm{~g})$ & 8872 & Mutillidae & - & 39 \\
\hline Platygastridae & 65 & 3160 & Pompilidae & 28 & 283 \\
\hline CYNIPOIDEA & & & Sapygidae & 2 & 29 \\
\hline Cynipidae & 41 & 186 & Scoliidae & 1 & 1 \\
\hline Figitidae & 58 & 1271 & Tiphiidae & 1 & 48 \\
\hline CHALCIDOIDEA & & & APOIDEA & & \\
\hline Aphelinidae & 31 & 419 & Vespidae & 24 & 259 \\
\hline Chalcididae & 18 & 170 & Apidae & $28(\mathrm{~g})$ & 6014 \\
\hline Elasmidae & 4 & 28 & Sphecidae & 107 & 1601 \\
\hline Total Hymenoptera & & & & 1310 & 83668 \\
\hline
\end{tabular}

ro; por último, 11 familias representadas en las muestras (las que figuran en la tabla sólo con el dato del número de ejemplares colectado) permanecen aún sin identificar. Hay que resaltar, sin embargo, que entre las familias identificadas están representadas todas las superfamilias importantes y grupos tróficos de Hymenoptera.

El total de especies identificadas hasta el momento asciende a 1310. En general las familias más abundantes resultan ser también las más ricas en especies. Hay que resaltar el predominio de las especies de familias con modo de vida parasitoide, destacando Pteromalidae con 290 especies, Ichneumonidae con 217 y Sphecidae y Eulophidae con 107 y 101 especies, respectivamente.

\section{NOVEDADES TAXONÓMICAS Y FAUNÍSTICAS}

Como cabía esperar las novedades taxonómicas y faunísticas de este estudio han sido muy numerosas. Éste es precisamente uno de los objetivos principales de un inventario de fauna. La sierra de Guadarrama es una de las áreas geográficas de 
España más estudiadas y mejor conocidas en cuanto a su fauna entomológica. En efecto, la historia del estudio de la sierra de Guadarrama, especialmente la vertiente madrileña, por su cercanía a Madrid, es larga y fructífera. Las prospecciones y muestreos en la sierra comenzaron ya con los albores del siglo XX por parte de figuras históricas de la entomología española radicados en el Museo Nacional de Ciencias Naturales. Más adelante, y en tiempos más recientes, se profundizó en el estudio entomológico de la sierra de Guadarrama fundamentalmente con trabajos realizados por entomólogos del Instituto español de entomología así como con varias tesis doctorales realizadas en las universidades madrileñas. A pesar de todo ello el conocimiento de la entomofauna de la sierra madrileña sigue siendo notoriamente insuficiente como revelan las novedades del presente estudio.

Aparte del hecho notable de que por primera vez en la entomología española se citan casi más de 1300 especies de himenópteros de un solo lugar de muestreo, el inventario ha dado como resultado otras novedades taxonómico-faunísticas. El número de especies nuevas para la ciencia formalmente descritas con material procedente del presente estudio es de 10: 7 especies nuevas de Platygastridae (Buhl, 2001), 2 de Chalcididae, incluyendo la primera cita en Europa del género Tanycoryphus (Rasplus \& Delvare, 1996; Delvare et al., 1999) y una de Ichneumonidae (Kolarov, 1996). Por otra parte, se han detectado nuevas especies, aún en espera de descripción, entre el material identificado de las familias Ichneumonidae, Figitidae, Pteromalidae Eulophidae y Aphelinidae. Es previsible que el número de nuevas especies se incremente notablemente cuando se concluya el estudio de todas las familias, especialmente entre los Proctotrupoidea y Chalcidoidea que son los grupos de himenópteros peor conocidos y estudiados.

El número de primeras citas para la Península Ibérica entre los táxones de Hymenoptera inventariados no puede aún evaluarse en su totalidad dado que no existen catálogos actualizados de todas las familias de himenópteros ibéricas, pero es, en cualquier caso, muy numeroso. Se citan 24 géneros y 123 especies nuevas de Pteromalidae (Garrido \& NievesAldrey, 1999), 33 especies nuevas de Platygastridae (Buhl \& Nieves-Aldrey, 2001), 1 género y 3 especies de Chalcididae (Pujade-Villar et al., 1998), 1 género y especie de Cynipidae (Nieves-Aldrey, 1995), 1 especie de afiidino (Braconidae) (Sanchis et al., 1995), 7 nuevos géneros de eucoilinos (Cynipoidea, Figitidae) (Fontal-Cazalla, 2001), varios géneros y numerosas especies de Eulophidae (Askew \&
Nieves-Aldrey, datos inéditos), además de un nuevo género para Europa) (Viggiani \& Nieves-Aldrey, 1993). Otras novedades para la fauna ibérica detectadas en las restantes familias inventariadas no han sido aún cuantificadas con precisión.

\section{Discusión}

ESTIMACIÓN DE LA RIQUEZA TOTAL DE HYMENOPTERA Y DE INSECTA EN EL ÁREA ESTUDIADA

La pregunta más inmediata a la vista del análisis de los datos del inventario es ¿cuántas especies de Hymenoptera y, más aún, de total de Insecta alberga el área estudiada? Esta cuestión era, en última instancia, uno de los principales objetivos del inventario. El número de especies de las familias de himenópteros encontradas en el área, pero aún no identificadas, puede ser estimado por extrapolación a partir de los datos de la riqueza total conocida de dichas familias en la Península Ibérica y el de la de las familias ya identificadas en el inventario. La media del porcentaje de riqueza que sobre el total ibérico presentan las familias identificadas puede ser aplicada a las familias aún no identificadas, pero colectadas en abundancia en el inventario; obtendremos, de este modo sus cifras estimadas de riqueza en el área estudiada. Las cifras calculadas se reflejan en la tabla III. El cómputo total de riqueza de Hymenoptera en el área de El Ventorrillo da un resultado de 2709 especies. Este número puede tener un relativamente alto margen de error debido al cálculo de la extrapolación. Sin embargo consideramos que la cifra obtenida es una subestimación de la riqueza total de Hymenoptera en el área, dado que los números reales de especies de las familias identificadas serán mayores que los obtenidos en el total de muestreos realizados, considerando que en casi todas las familias analizadas no se ha alcanzado la asíntota en las respectivas curvas de acumulación de especies, con relación a la intensidad de los muestreos.

Hymenoptera, junto a Coleoptera, Diptera y Lepidoptera es uno de los cuatro grandes grupos hiperdiversos de insectos. Se ha estimado en 47.000 el número total de especies de insectos en la Península Ibérica (Martín-Piera \& Lobo, 2000; Ramos et al., 2001). Los himenópteros con unas 9.800 especies suponen un $20,6 \%$ del total de la riqueza ibérica de insectos (Martín-Piera \& Lobo, 2000; Ortuño, 2002; Nieves-Aldrey, datos inéditos). Si este mismo porcentaje se aplica a la muestra inventariada, el número estimado total de insectos en el área de El Ventorrillo sería de más de 13.000 especies. A pesar de que, a priori, esta cifra 
Tabla III.- Riqueza en especies de familias de Hymenoptera en el inventario de "El Ventorrillo" comparada con datos similares de otros inventarios de España, Canadá y Holanda. Se incluyen cifras estimadas de riqueza global de familias de Hymenoptera de España y el conjunto mundial. De izquierda a derecha, datos de Nieves-Aldrey et al (este trabajo), Melic \& Blasco-Zumeta (1999), Finnamore (1994), Zuilgen et al, (1996), Nieves-Aldrey (inédito) y Nieves-Aldrey \& Fontal-Cazalla (1999). Las cifras de la primera columna, a la izquierda, corresponden a las especies identificadas hasta la fecha (las familias identificadas al 100\% de las muestras figuran en negrita). La segunda columna a la izquierda representa las especies estimadas en las muestras colectadas de familias aún no identificadas, cifras estimadas por extrapolación de las especies estimadas para el conjunto de España (más explicación en el texto).

Table III.- Species-richness of families of Hymenoptera from the inventory of "El Ventorrillo" and its comparison with similar data from other site inventories from Spain, Canada and Nederland. Estimates on global species-richness of Hymenoptera from Spain, and the World are also indicated. From left to right, data from Nieves-Aldrey et al. (present data), Melic \& Blasco-Zumeta, (1999), Finnamore (1994), Zuilgen et al. (1996), Nieves-Aldrey (unpublished) and Nieves-Aldrey \& Fontal-Cazalla (1999). Numbers on the first column (on the left) correspond to the identified species until now (numbers of families fully identified figure in bold).The second column on the left represent the estimated species from families with collected samples but not yet identified, numbers calculated for extrapolation from numbers of species-richness of hymenopteran families in the whole Spain (for more explanation see text).

\begin{tabular}{|c|c|c|c|c|c|c|c|}
\hline $\begin{array}{l}\text { Familias de Hymenoptera } \\
\text { (número de especies) }\end{array}$ & $\begin{array}{l}\text { EI Ventorr } \\
\text { ident. }\end{array}$ & $\begin{array}{l}\text { illo (España) } \\
\text { Estim. }\end{array}$ & Monegros (España) & Wagner fen (Canadá) & De Brand (Holanda) & España & Mundo \\
\hline Xyelidae & 2 & & - & - & - & 6 & 56 \\
\hline Megalodontidae & 2 & & 1 & - & - & 10 & 70 \\
\hline Pamphiliidae & - & - & - & 1 & - & 30 & 232 \\
\hline Argidae & 5 & & - & 2 & 1 & 25 & 812 \\
\hline Blasticotomidae & - & - & - & - & - & 1 & 9 \\
\hline Cimbicidae & 4 & & 1 & 2 & - & 25 & 131 \\
\hline Diprionidae & - & - & - & - & - & 12 & 90 \\
\hline Tenthredinidae & 98 & & 8 & 78 & 100 & 500 & 3840 \\
\hline Cephidae & 7 & & 2 & 1 & 2 & 25 & 79 \\
\hline Siricidae & - & - & - & - & - & 5 & 87 \\
\hline Xiphydriidae & - & - & - & - & - & 4 & 94 \\
\hline Orussidae & 1 & & - & - & - & 4 & 69 \\
\hline Stephanidae & - & - & - & - & - & 2 & 100 \\
\hline Trigonalyidae & - & - & - & - & 1 & 1 & 75 \\
\hline Aulacidae & - & - & - & 1 & - & 5 & 150 \\
\hline Evaniidae & 1 & & - & - & - & 5 & 400 \\
\hline Gasteruptiidae & - & 5 & - & 2 & 2 & 20 & 500 \\
\hline Ceraphronidae & - & 11 & 8 & 14 & 1 & 40 & 354 \\
\hline Megaspilidae & - & 19 & 7 & 6 & 9 & 70 & 448 \\
\hline Heloridae & 1 & & 1 & 1 & 1 & 3 & 7 \\
\hline Diapriidae & - & 98 & 1 & 55 & 19 & 350 & 2300 \\
\hline Proctotrupidae & - & 14 & 1 & 7 & - & 50 & 310 \\
\hline Scelionidae & 14(g) & 36 & 1 & 28 & 6 & 130 & 1100 \\
\hline Platygastridae & 65 & & 35 & 35 & 10 & 200 & 3000 \\
\hline Cynipidae & 41 & & 4 & 1 & 11 & 140 & 1369 \\
\hline Figitidae & 58 & & 6 & 57 & 21 & 150 & 1411 \\
\hline Ibaliidae & - & & - & - & - & 1 & 20 \\
\hline Agaonidae & - & - & - & - & - & 1 & 650 \\
\hline Aphelinidae & 31 & & 19 & 2 & 3 & 70 & 1120 \\
\hline Chalcididae & 18 & & 23 & - & - & 50 & 1875 \\
\hline Elasmidae & 4 & & 8 & - & - & 9 & 260 \\
\hline Encyrtidae & 19 & 98 & 55 & 38 & 8 & 350 & 3825 \\
\hline Eucharitidae & - & - & 1 & - & - & 5 & 380 \\
\hline Eulophidae & 101 & 126 & 80 & - & 44 & 450 & 3900 \\
\hline Eupelmidae & 10 & & 26 & - & 2 & 50 & 900 \\
\hline Eurytomidae & - & 33 & 9 & - & - & 120 & 1425 \\
\hline Leucospidae & 2 & & 1 & - & - & 6 & 240 \\
\hline Mymaridae & $2 \mathrm{~g}$ & 28 & 3 & 33 & 11 & 100 & 1400 \\
\hline Mymarommatidae & - & - & 1 & - & - & 1 & 9 \\
\hline Ormyridae & 4 & & 7 & - & - & 10 & 90 \\
\hline Perilampidae & - & 5 & 8 & 1 & - & 20 & 260 \\
\hline Pteromalidae & 290 & & 123 & 40 & 52 & 700 & 4115 \\
\hline Signiphoridae & 1 & & 4 & - & - & 4 & 80 \\
\hline Tetracampidae & 2 & & 3 & - & 1 & 8 & 50 \\
\hline Torymidae & - & 28 & 22 & - & 11 & 100 & 1150 \\
\hline Trichogrammatidae & 1 & 14 & 1 & - & - & 50 & 675 \\
\hline Braconidae & 26 & 364 & 9 & 248 & 278 & 1300 & 40000 \\
\hline Ichneumonidae & 217 & 644 & 37 & 665 & 92 & 2300 & 60000 \\
\hline Bethylidae & - & 14 & 16 & 4 & - & 50 & 2200 \\
\hline Chrysididae & 43 & & 42 & 7 & 5 & 170 & 3000 \\
\hline Dryinidae & 20 & & 14 & 8 & 13 & 40 & 1100 \\
\hline Embolemidae & 1 & & 1 & - & - & 1 & 10 \\
\hline Formicidae & 28 & & 53 & 9 & 8 & 240 & 8800 \\
\hline Mutillidae & - & 14 & 15 & - & - & 52 & 5000 \\
\hline Pompilidae & 28 & & - & 14 & 9 & 200 & 4200 \\
\hline Sapygidae & 2 & & - & 1 & - & 7 & 80 \\
\hline Scoliidae & $\overline{1}$ & & 5 & - & - & 25 & 300 \\
\hline Tiphiidae & 1 & 5 & - & - & - & 20 & 1500 \\
\hline Vespidae & 24 & & 66 & 14 & 12 & 70 & 4170 \\
\hline Apidae & $28(\mathrm{~g})$ & 252 & 97 & 51 & 19 & 900 & 20000 \\
\hline Sphecidae & 107 & & 2 & 25 & 45 & 500 & 8000 \\
\hline Otras 20 familias & - & - & - & - & - & - & 1027 \\
\hline TOTALES & 1310 & 2709 & 821 & 1410 & 799 & 9790 & 199000 \\
\hline
\end{tabular}


pueda ser considerada muy alta, la consideramos razonable a la vista de los resultados del inventario y es, en cualquier caso, una hipótesis de trabajo que puede ser o no refutada con los datos aportados en la profundización ulterior y extensión del inventario a otros grupos de insectos.

\section{COMPARACIÓN CON DATOS DE OTROS INVENTARIOS}

Si bien son crecientes los proyectos actualmente en marcha que contemplan inventarios totales de biota o de entomofauna en distintas partes del globo (los ejemplos más conspicuos son en Costa Rica, Islas Hawaii y Smoky Mountains, EEUU, y Colombia) los resultados publicados de inventarios de insectos ya concluidos son muy escasos en la literatura científica. Sin embargo hay algunos ejemplos recientes de distintos países, basados en su mayor parte en programas de muestreo con trampas Malaise. Unos pocos se han centrado fundamentalmente, al igual que el nuestro, en el orden Hymenoptera, o bien presentan resultados completos de este orden, lo que facilita la comparación directa y permite extraer conclusiones de los resultados respectivos de los inventarios.

En el ámbito internacional hemos elegido dos trabajos realizados, uno en Europa, en una reserva natural de Holanda, integrada por bosques, marismas y pastos húmedos (Zuilgen et al. (1996) y el otro en América del Norte, en el Área Natural Wagner, un área pantanosa en la provincia de Alberta (Canadá) (Finnamore, 1994). En la Península Ibérica el único ejemplo existente es el muy completo inventario realizado en Retuerta de Pina (Zaragoza), un sabinar enclavado en la amplia región árida de Los Monegros (Melic \& Blasco Zumeta, 1999). Las cifras comparativas se reflejan en la tabla III.

La riqueza de Hymenoptera, y la estimada total de Insecta, de la Estación de El Ventorrillo supera ampliamente las de otras áreas inventariadas publicadas en la literatura. La cifra de 1410 especies de Hymenoptera del pantano canadiense, grupo identificado en su totalidad, es cercana a la de 1310 especies identificadas en El Ventorrillo hasta el momento, pero está lejana a las 2709 especies estimadas como riqueza total del orden en el área de la Estación Biológica. Por otra parte, la diversidad de Hymenoptera en aquella zona está singularizada fundamentalmente por la superfamilia Ichneumo-noidea, en especial por la familia Ichneumonidae con 665 especies, más 248 especies de Braconidae, que en conjunto suponen casi el $65 \%$ del total. Por lo que se refiere al inventario del área natural holandesa, no obstante ser un área mucho más extensa que El
Ventorrillo, presenta unas cifras globales de Hymenoptera (799 especies), así como parciales de familias, notablemente más bajas, con excepción de las familias Tenthredinidae y Braconidae, grupos cuyo centro de diversidad se encuentra en latitudes semiboreales.

El por muchos conceptos admirable, minucioso, y casi completo inventario llevado a cabo por Javier Blasco Zumeta en los Monegros registra 821 especies de himenópteros, cifra notable tratándose de una zona árida, cuyo valor reside más que por la cifra en sí por el elevado número de novedades taxonomico-faunísticas que registra, con profusión de endemismos, novedades que, por otra parte, entraba dentro de lo previsible encontrar dada la singularidad del enclave geográfico, casi un desierto a las puertas de la Europa atlántica, y muy escasos o nulos antecedentes de estudio para la mayoría de los grupos de insectos. La composición porcentual de la riqueza de himenópteros en Retuerta de Pina es muy diferente a la de El Ventorrillo y está dominada por especies de Chalcidoidea, singularmente Pteromalidae y Eulophidae, con alta representación de otros himenópteros de hábitos termófilos como los Chrysididae. Por el contrario, familias que en El Ventorrillo son mayoritarias como Ichneumonidae y Tenthredinidae son muy escasas en el inventario de Retuerta de Pina.

\section{Conclusiones}

- La estación Biogeológica de El Ventorrillo, en el sector central de la Sierra de Guadarrama, alberga una extraordinaria diversidad entomológica, como indican los resultados del inventario de insectos llevado a cabo a lo largo de varios años en la zona empleando distintos métodos de muestreo, fundamentalmente trampas Malaise. El registro de las colectas con trampa Malaise indica abundancias y riquezas muy altas de las poblaciones de insectos, comparativamente más altas que las medidas en otros inventarios similares tanto de zonas templadas como tropicales. Se han capturado, separado e identificado hasta el nivel de orden 581.000 ejemplares de insectos, de los cuales cerca de 84.000 correspondieron a Hymenoptera. Las especies identificadas de este orden hasta el momento son 1.310, con una riqueza total de himenópteros estimada en más de 2.700 especies y de 13.000 para el conjunto de la Clase Hexapoda. 
- Los datos del presente inventario son un registro de la Biodiversidad actual del área de la Sierra de Guadarrama estudiada, que queda archivado y salvaguardado para la posteridad en las colecciones científicas del Museo Nacional de Ciencias Naturales, las cuales se han enriquecido notablemente con los cientos de miles de ejemplares y los miles de especies colectados en el proyecto.

- La rica Biodiversidad del inventario de El Ventorrillo, sin parangón con la conocida de otras áreas, a partir de datos publicados de inventarios similares, se registra paradójicamente en un área semi-natural, modelada por una antigua interacción humana con el paisaje y con una muy fuerte y reciente presión humana y urbanística por la relativa vecindad de la urbe de Madrid y la cercanía de los municipios de Cercedilla y Navacerrada y el Puerto de Navacerrada, por el que transitan cada año cientos de miles de visitantes. Se pone de manifiesto que altas tasas de Biodiversidad son compatibles con el uso tradicional del territorio en una naturaleza, que en la cuenca mediterránea, interactúa y está en frágil equilibrio con el hombre desde tiempos remotos. La reciente propuesta de creación del Parque Nacional de la Sierra de Guadarrama puede preservar esta riqueza biológica en un área cada vez más sometida a fuerte presión antropógena. Se insta a los responsables políticos a acelerar los trámites para salvaguardar la Biodiversidad privilegiada de esta área de la Sierra de Guadarrama y de la Comunidad madrileña.

- El inventario biológico completado es un instrumento precioso para el análisis futuro de la Biodiversidad del área estudiada, permitiendo registrar y valorar los posibles cambios por alteraciones naturales (calentamiento global) o inducidos por el hombre (destrucción o alteración del hábitat).

\section{AGRADECIMIENTOS}

Este trabajo no se hubiera podido llevar a cabo sin la generosa ayuda prestada por un numeroso equipo internacional de especialistas en Hymenoptera que colaboraron desinteresadamente en la identificación de muestras de distintas familias. Expresamos nuestra gratitud a Dick Askew (Eulophidae y Pteromalidae (en parte); L. Scaramozzino, R. Hinz, J. Kolarov, K. Hortssmann, J. P. Aeschlimann, C. Thirion y J. Selfa (Ichneumonidae); G. Delvare y J. Pujade (Chalcididae); G. Gibson (Eupelmidae); M. Olmi (Dryinidae); G. Llorente, W. Schedl y D. Sheppard (Symphyta); G. Viggiani (Aphelinidae);
J. Noyes (Encyrtidae); S. F. Gayubo (Sphecidae); J. Gusenleitner y L. Castro (Sapygidae y Vespidae); T. Osten (Scoliidae); J. Serrano (Formicidae); V. Caleca (Scelionidae); E. Mingo (Chrysididae); F. J. Ortiz Sanchez (Apidae); J. Abenius (Pompilidae); F. Sanza (Eumenidae). Un agradecimiento muy especial merece Bernardino Torres, veterano trabajador de la Estación Biogeológica de El Ventorrillo por su valiosa ayuda en la instalación y mantenimiento de las trampas Malaise. Por último, aunque no en menor medida, expresamos nuestra gratitud a los muchos colegas, auxiliares de investigación y estudiantes del Museo Nacional de Ciencias Naturales que ayudaron en la penosa e ingrata tarea de separación de las muestras de las trampas Malaise. Un evaluador anónimo señaló errores y aportó valiosas sugerencias para mejorar el manuscrito. El trabajo fue financiado con fondos de la consejería de educación de la Comunidad de Madrid, proyecto C188/91, así como, parcialmente, con fondos de la DGES PB97-1241, y REN2002-03518 concedidos a J.L.N.A.

\section{Referencias}

Allué Andrade, J. L., 1987. Memoria del mapa de subregiones fitoclimáticas. In: Rivas Martinez, S., Memoria del mapa de series de vegetación de España (1:400.000). Ministerio de Agricultura, Pesca y Alimentación (ICONA). Madrid: 221-223.

Balleto, E. \& Casale, A., 1991. Mediterranean insect conservation. In: N. M. Collins \& J. A. Thomas (eds). The conservation of insects and their habitats. Academic Press. London: 121-140.

Buhl, P. N., \& Nieves-Aldrey, J. L. 2001. Taxocenosis of platygastrids in two habitats in central Spain (Hymenoptera, Platygastridae). Frustula Entomologica, XXIII (2000): 142-160.

Colwell, R. K. \& Coddington, J. A., 1994. Estimating terrestrial biodiversity through extrapolation. Philosophical Transactions of the Royal Society of London, B, 345: 101-118

DARling, D. C. \& PACKer, L., 1988. Effectiveness of Malaise traps in collecting Hymenoptera: The influence of trap design, mesh size, and location. Canadian Entomologist, 120: 787-790.

Delvare, G., Pujade-Villar, J. \& Rasplus, J. I. 1999. Revision des Belaspidia ouest-paléarctiques (Hymenoptera: Chalcididae). Annales de la Societé entomologique de France, 35(3-4): 329-345

Finnamore, A. T., 1994. Hymenoptera of the Wagner natural area, a boreal spring fen in central Alberta. Memoirs of the Entomological Society of Canada, 169: 181-220.

Fontal-Cazalla, F. M., 2001. Filogenia y diversidad de eucoilinos paleárticos y neotropicales. Universidad Autónoma de Madrid. Tesis doctoral inédita. 495 pp.

García-Barros, E., Gurrea, P., Luciáñez, M. J., Martín-Cano, J., Munguira, M. L., Moreno, J. C., Sainz, H., SAnz, M. J. \& Simón, J. C., 2002. Parsimony analysis of endemicity and its application 
to animal and plant geographical distributions in the Ibero-Balearic region (western Mediterranean). Journal of Biogeography, 29(1): 109-124.

Garrido, A. M. \& Nieves-Aldrey, J. L., 1992. Estructura y dinámica de una taxocenosis de Pteromalidae (Hymenoptera, Chalcidoidea) en el sector medio de la Sierra de Guadarrama. Eos, 68(1): 29-49.

Garrido Torres, A. \& Nieves-Aldrey, J. L. 1999. Pteromálidos de la Comunidad de Madrid: faunística y catálogo. Graellsia, 55: 9-147.

Gaston, K. J., 1993. Spatial Patters in the Description and Richness of the Hymenoptera. In: J. Lasalle \& I. D. Gauld (eds.). Hymenoptera and Biodiversity. C.A.B. International. Wallingford: 277-293.

GASTON, K. J., 1996. Species richness: measure and measurement. In: K. J. Gaston (ed.). Biodiversity. A Biology of Numbers and Difference. Blackwell Science. Oxford: 77-113.

Gaston, K. J., 2000. Global patterns in biodiversity. Nature, 405: 220-226.

Gaston, K. J., Gauld, I. D. \& Hanson, P., 1996. The size and composition of the hymenopteran fauna of Costa Rica. Journal of Biogeography, 23: 105-113.

Gauld, I. \& Hanson, P. E., 1995. The order Hymenoptera. In: P. E. Hanson \& I. Gauld (eds). The Hymenoptera of Costa Rica. The Natural History Museum. London: 4-6.

Godfray, H. C. J., Lewis, O. T. \& Memmott, J. 1999. Studying insect diversity in the tropics. Philosophical Transactions of the Royal Society of London, B, 354: 1811-1824.

HAMmOND, P. M., 1990. Insect abundance and diversity in the Dumoga-Bone National Park, North Sulawesi, with special reference to the beetle fauna of lowland rainforest in the Toraut region. In: W. J. Knight \& J. D. Holloway (eds.). Insects and the Rain Forests of South East Asia (Wallacea). The Royal Entomological Society of London. London: 197-254.

Holden, C., 1989. Entomologists wane as insects wax. Science, 246: 754-756.

Kolarov, J., 1996. A study of Spanish Cremastinae (Hymenoptera, Ichneumonidae). Linzer Biologische Beiträge, 28(2): 821-827.

LaSalle, J. \& Gauld, I. D. (eds). 1993. Hymenoptera and Biodiversity. C.A.B. International. Wallingford. 348 pp.

LaSalle, J. \& Gauld, I. D., 1991. Parasitic Hymenoptera and the biodiversity crisis. Redia, 74(3): 315-334.

Lobo, J. M., Castro, I. \& Moreno, J. C., 2001. Spatial and environmental determinants of vascular plant species richness distribution in the Iberian Peninsula and Balearic Islands. Biological Journal of the Linnaean Society, 73: 233-253.
Lobo, J. M. \& Martín-Piera, F., 2002. Searching for a predictive model for species richness of Iberian dung beetle based on spatial and environmental variables. Conservation Biology, 16(1): 158-173.

Lockwood, J. A., Shaw, S. R. \& Struttmann, J. M., 1996. Biodiversity of wasp species (Insecta: Hymenoptera) in burned and unburned habitats of Yellowstone National Park, Wyoming, USA. Journal of Hymenoptera Research, 5: 1-15.

Martín, J. \& Gurrea, P., 1999. Areas de especiación en España y Portugal. Boletín de la Asociación española de Entomología, 23: 83-103.

Martín-Piera, F., 2001. Area networks for conserving Iberian insects: A case study of duna beetles (col., Scarabaeoidea). Journal of Insect Conservation, 5: 233-252.

Martín-Piera, F. \& Lobo, J. M., 2000. Diagnóstico sobre el conocimiento sistemático y biogeográfico de tres órdenes de insectos hiperdiversos en España: Coleoptera, Hymenoptera y Lepidoptera. In: F. Martín-Piera, J.J. Morrone \& A. Melic (eds). Hacia un proyecto CYTED para el Inventario y Estimación de la Diversidad Biológica en Iberoamérica. PrIBES2000. Monografías Tercer Milenio. Sociedad entomológica aragonesa (SEA). Zaragoza: 287-308.

MartínEZ DE MurguíA, L., 2002. La taxocenosis de Hymenoptera en Artikutza (Navarra). Boletín de la Sociedad entomológica Aragonesa, 31: 227-237.

Matthews, R. H. \&. Matthews, J. R., 1970. The Malaise trap: its utility and potential for sampling insect populations. The Michigan entomologist, 4(4): 117-122.

MAY, R. M., 1988. How many species are there on Earth? Science, 241: 1441-1449.

Melic, A. \& Blasco-Zumeta, J. (eds). 1999. Manifiesto Científico por los Monegros. Volumen Monográfico. Boletín de la S.E.A., 24 (1998). 266 pp.

Myers, N., \& Cowling, R.M., 1999. Mediterranean Basin. In: R. A. Mittermeier, N. Meyers, P. Robles Gil \& C. G. Mittermeier (eds.). Hotspots: Earth's Biologically Richest and Most Endagered Terrestrial Ecoregions. CEMEX. Mexico: 254-267.

Myers, N., Mittermeier, R. A., Mittermeier, C. G., Da Fonseca, G. A. B. \& Kents, J., 2000. Biodiversity hotspots for conservation priorities. Nature, 403: 853-858.

NiEves-AldRey, J. L., 1995. Abundancia, diversidad y dinámica temporal de cinípidos en dos hábitats del centro de España (Hymenoptera, Cynipidae). In: Comité organizador del VI Congreso Iberico de Entomología (eds.). Avances de Entomología Ibérica. Museo Nacional de Ciencias Naturales (CSIC) y Universidad Autónoma de Madrid. Madrid: 113-136.

Nieves-Aldrey, J. L. \& Fontal-Cazalla, F., 1997. Los insectos de la isla de Coiba (Panamá). Abundancia y dinámica estacional. Análisis del caso de los hime- 
nópteros (Hexapoda Hymenoptera). In: Santiago Castroviejo (ed.). Flora y Fauna del Parque Nacional de Coiba (Panamá) Inventario preliminar. A.E.C.I. Madrid: 329-361.

Nieves-Aldrey, J. L. \& Fontal-Cazalla, F., 1999. Filogenia y Evolución del Orden Hymenoptera. In: Melic, A. (ed.). Evolución y Filogenia de Arthropoda. Volumen Monográfico de la S.E.A. Zaragoza: 459474.

Nieves-Aldrey, J. L \& Rey Del Castillo, C., 1991. Ensayo preliminar sobre la captura de insectos por medio de una trampa "Malaise" en Sierra de Guadarrama (España) con especial referencia a los himenópteros. Ecología, 5: 383-403.

Noyes, J. S., 1982. Collecting and preserving chalcid wasps. Journal of Natural History, 16(3): 315-334.

Noyes, J. S., 1989a. A study of five methods of sampling Hymenoptera (Insecta) in a tropical rain forest, with special reference to the Parasitica. Journal of Natural History, 23: 285-298.

NOYES, J. S., 1989b. The diversity of Hymenoptera in the tropics with special reference to Parasitica in Sulawesi. Ecological Entomology, 14: 197-207.

Ortuño, V. M., 2002. Estado de conocimiento de los artrópodos de España. In: Díaz Pineda, F. et al. (eds.). La Diversidad Biológica de España. CYTED. Prentice Hall. Madrid: 209-234.

Owen, D., 1983. A hole in a tent or how to explore insect abundance and diversity. Contributions of the America Entomological Institute, 20: 32-46.

Pujade-Villar, J., 1996. Resultados preliminares obtenidos a partir de una trampa Malaise situada en una zona mediterránea pirenaica. Pirineos, 147-148: 61-80.

Pujade-Villar, J., Nieves-Aldrey, J. L. \& Delvare, G., 1998. Taxocenosis de calcídidos en dos hábitats bien conservados del centro de España (Hym., Chalcidoidea, Chalcididae). Boletín de la Real Sociedad española de Historia Natural (Sección Biológica), 94 (3-4): 87-96.

Purvis, A. \& Hector, A., 2000. Getting the measure of biodiversity. Nature, 405: 212-219.

Ramos, M. A., Lobo, J. M. \& Esteban, M. 2001. Ten years inventorying the Iberian Fauna: results and perspectives. Biodiversity and Conservation, 10: 19-28.

Rasplus, J. I. \& Delvare, G., 1996. Revision des Tanycoryphus (Hymenoptera: Chalcididae) ouestPalearctiques et description de deux nouvelles especes europeennes. Annales de la Societé entomologique de France, 32(2): 223-232.

Rivas Martínez, S., 1982. Memoria del mapa de las series de vegetación de la provincia de Madrid (1:200.000). Diputación de Madrid, Servicio Forestal y Medio Ambiente. Madrid. 48 pp.
Sanchis, A., Michelena, J. M., Nieves-Aldrey, J. L. \& Rey DEL CAstillo, C., 1995. Afidiinos (Hymenoptera: Braconidae, Aphidiinae) del centro peninsular. Boletín de la Asociación española de Entomología, 19(1-2): 219-228.

Segade, C., Ros-Farre, P. A., Ventura, D. \& PujadeVILlAR, J., 1997. Estudio comparativo de las capturas realizadas con trampa Malaise en Andorra con especial atencion a los himenópteros (Hymenoptera). Zapateri, 7: 71-82.

Siemann, E., Tilman, D., HaArstad, J. \& Ritchie, M., 1998. Experimental tests of the dependence of arthropod diversity on plant diversity. American Naturalist, 152: 738-750.

Stork, N. E., Samways, M. J. \& Eeeley, H. A. C., 1996. Inventorying and monitoring biodiversity. Trends in Ecology and Evolution, 11: 39-40.

Tilman, D., 2000. Causes, consequences and ethics of biodiversity. Nature, 405: 208-211.

Tomé, M. A. M., González, J. A., Gayubo, S. F. \& TORRES, F., 2001. Estudio comparativo sobre la eficiencia de captura de insectos (Arthropoda, Hexapoda) mediante trampas Malaise, en un biotopo arenoso de la submeseta Norte (España). Boletín de la Real Sociedad española de Historia Natural (Sección Biológica), 96(3-4): 231-241.

Townes, A., 1972. A light-weigth Malaise trap. Entomological News, 83: 239-247.

Ulrich, W., 1999. The number of species of Hymenoptera in Europe and assesment of the total number of Hymenoptera in the world. Polskie Pismo Entomologiczne, 68: 151-164.

Viggiani, J. L. \& Nieves-Aldrey, J. L. 1993. Prima segnalazione di Goetheana shakespearei Girault (Hymenoptera Eulophidae), parasitoide esotico di Thysanoptera, per l'Europa. Bollettino di Zoología Agraria e di Bachicoltura, Ser. II, 25(1): 105-108.

Yoon, C. K., 1993. Counting creatures great and small. Science, 260: 620-622.

Zuilgen, J. W. van, Peeters, T., Wielink, P. van, Eck, A. VAN \& Bouvy, E. (eds.), 1996. Brand-Stof. Een inventarisatie van de entomofauna van het natuurreservaat 'De Brand'in 1990. KNNV. Insektenwerkgroep. Afdeling Tilburg. 228 pp. 
Apéndice.- Se da a continuación el listado alfabético de Hymenoptera, en el inventario de El Ventorrillo. Al lado de cada especie figura, entre paréntesis, el total de ejemplares colectado en los muestreos; en la familia Cynipidae las especies seguidas de un asterisco señalan especies cuya presencia en el área consta por observación de su agalla y no por ejemplares colectados. Los taxones identificados solamente hasta el nivel de subfamilia, tribu o género se incluyen también en la lista, juntamente con la cifra correspondiente del total de ejemplares colectado.

Appendix. - The alphabetic list of Hymenoptera inventoried from El Ventorrillo is given below. Numbers in brackets, right to each taxon name, correspond to total specimens collected in samplings. In the case of the family Cynipidae an asterisk indicate the species whose presence is verify by their galls but nor for adult collected specimens. Taxa identified to subfamily, tribu or genus level only are also included in the list, jointly the numbers corresponding total specimens collected.

\begin{tabular}{|c|c|}
\hline APHELINIDAE & Chelostoma (12) \\
\hline Aphelinus sp 1 (1) & Heriades $(5)$ \\
\hline Aphelinus sp 2 (27) & Hoplitis (68) \\
\hline Aphelinus sp 3 (14) & Lithurgus (1) \\
\hline Aphelinus sp 4 (1) & Megachile (28) \\
\hline Aphelinus sp 5 (1) & Osmia (71) \\
\hline Aphelinus spp (120) & Stelis (4) \\
\hline Aphelinus (M) subflavescens (Westwood, 1837) (3) & Melittinae \\
\hline Aphelinus (Mesidiopsis) sp (4) & Dasypoda (3) \\
\hline Aphytis sp 1 (2) & ARGIDAE \\
\hline Aphytis sp 2 (6) & Arge cyanocrocea (Förster, 1771) (2) \\
\hline Centrodora sp 1 (4) & Arge melanochroa (Gmelin, 1790) (4) \\
\hline Centrodora sp 2 (2) & Arge nigripes (Retzius, 1783) (4) \\
\hline Centrodora sp 3 (1) & Sterictiphora gastrica (Klug, 1812) (9) \\
\hline Centrodora sp 4 (17) & Sterictiphora geminata (Gmelin, 1790) (10) \\
\hline $\begin{array}{l}\text { Centrodora sp } 5(11) \\
\text { Coccophagus sp }(9)\end{array}$ & BRACONIDAE \\
\hline Encarsia cfr aspidioticola (Mercet, 1929) (1) & Adeliinae (5) \\
\hline Encarsia sp "group aspidioticola" (1) & Agathidinae (7) \\
\hline Encarsia sp "group citrina" (1) & Alysiinae (333) \\
\hline Encarsia sp "group lutea" (2) & Aphidiinae \\
\hline Encarsia sp 1 "group partenopea" (11) & Adialytus ambiguus (Haliday,1834) (12) \\
\hline Encarsia sp 2 "group partenopea" (1) & Aphidius absinthii Marshall,1896 (1) \\
\hline Encarsia leucaspidis (Mercet, 1912) (3) & Aphidius avenae Haliday,1834 (4) \\
\hline Encarsia longicornis (Mercet, 1928) (9) & Aphidius eadyi Stary,González,Hall,1979 (4) \\
\hline Encarsia sp cfr longicornis Mercet (16) & Aphidius ervi Haliday, 1834 (109) \\
\hline Euderomphale sp. (1) & Aphidius matricariae Haliday,1834 (229) \\
\hline Marietta sp 1 (2) & Aphidius rhopalosiphi De Stefani,1902 (35) \\
\hline Marietta sp 2 (1) & Aphidius rosae Haliday, 1834 (29) \\
\hline Oligosita sp "group collina" (1) & Aphidius salicis Haliday,1834 (2) \\
\hline Pteroptrix bicolor (Howard, 1898) (2) & Aphidius sonchi Marshall,1896 (37) \\
\hline APIDAE & $\begin{array}{l}\text { Aphidius sp (16) } \\
\text { Aphidius urticae Haliday,1834 (12) }\end{array}$ \\
\hline Andreninae & $\begin{array}{l}\text { Aphidius urticae Haliday, } 1834 \text { (12) } \\
\text { Aphidius uzbekistanicus Luzhetzki,1960 (36) }\end{array}$ \\
\hline Andrena (446) & $\begin{array}{l}\text { Aphidius uzbekistanicus Luzhetzk1,1960 (36) } \\
\text { Diaeretiella rapae (Mc'Intosh,1855) (29) }\end{array}$ \\
\hline Panurgus (108) & Ephedrus persicae Froggatt,1904 (7) \\
\hline Antophorinae & Lipolexis gracilis Förster,1862 (2) \\
\hline Antophora (42) & Lysiphlebus fabarum (Marshall,1896) (17) \\
\hline Ceratina (224) & Monoctonus caricis (Haliday,1833) (5) \\
\hline Eucera (24) & Parapraon $s p(1)$ \\
\hline Heliophila (1) & Praon exsoletum $($ Nees, 1811$)$ \\
\hline Melecta (5) & Praon volucre (Haliday,1833) (3) \\
\hline Nomada (37) & Toxares deltiger Haliday,1833 (2) \\
\hline Tetralonia (1) & Trioxys angelicae (Haliday,1833) (3) \\
\hline Xylocopa (9) & Trioxys brevicornis (Haliday,1833) (7) \\
\hline Apinae & Trioxys pallidus (Haliday,1833) (6) \\
\hline Apis (8) & Trioxys $s p(1)$ \\
\hline Bombus (147) & Blacinae (677) \\
\hline Psithyrus (2) & Braconinae (22) \\
\hline Colletinae & Cheloninae (8) \\
\hline Colletes (8) & Doryctinae (12) \\
\hline Halictinae & Euphorinae (57) \\
\hline Dufourea (5) & Gnamptodontinae (6) \\
\hline Halictus (71) & Helconinae (6) \\
\hline Pseudapis (1) & Homolobinae (5) \\
\hline Sphecodes (975) & Ichneutinae (6) \\
\hline Megachilinae & Micrograstinae (154) \\
\hline Anthidium (74) & Miracinae (5) \\
\hline
\end{tabular}

Opiinae (57)

Rhogadiinae (16)

Braconidae indet. (10.353)

\section{CEPHIDAE}

Calameuta pallipes (Klug, 1803) (2)

Calameuta pygmaea (Poda) (1)

Cephus pygmeus (Linnaeus, 1767) (1)

Hartigia nigra (Harris, 1776) (4)

Hartigia sp (2)

Janus compressus (Fabricius, 1793) (3)

Trachelus tabidus (Fabricius, 1787) (3)

\section{CHALCIDIDAE}

Belaspidia obscura Masi,1916 (4)

Belaspidia elongata Pujade-Villar, 1999 (1)

Brachymeria femorata (Panzer,1801) (2)

Brachymeria inermis (Fonscolombe,1832) (19)

Brachymeria rugulosa (Forster,1859) (10)

Brachymeria secundaria (Ruschka,1932) (19)

Brachymeria tibialis (Walker,1834) (18)

Euchalcis unicolor (Lucas,1879) (4)

Haltichella rufipes (Olivier, 1790) (3)

Hockeria bifasciata Walker, 1834 (34)

Hockeria sp (1)

Hockeria unicolor Walker, 1834 (43)

Lasochalcidia pugnatrix Steffan,1958 (1)

Neochalcis fertoni (Kieffer, 1899) (9)

Proconura nigripes (Fonscolombe,1832) (1)

Psilochalcis benoisti (Steffan, 1948) (1)

Psilochalcis subarmata (Forster, 1859) (1)

Tanycoryphus ibericus Rasplus \& Delvare,1996 (2)

\section{CHRYSIDIDAE}

Chrysis angustula Schenck, 1856 (1)

Chrysis bidentata Linnaeus, 1767 (1)

Chrysis cortii Linsenmaier, 1951 (3)

Chrysis dusmetina Bohart, 1990 (1)

Chrysis emarginatula Spinola, 1808 (1)

Chrysis gracillima Förster, 1853 (8)

Chrysis grohmanni Dahlbom, 1854 (1)

Chrysis ignita (Linnaeus, 1758) (22)

Chrysis leachii Shuckard, 1836 (11)

Chrysis lusitanica Bischoff, 1910 (5)

Chrysis merceti (Trautmann, 1926) (1)

Chrysis ramburi Dahlbom, 1854 (1)

Chrysis rutilans Olivier, 1790 (1)

Chrysis scutellaris Fabricius, 1794 (4)

Chrysis semicincta Lepeletier, 1806 (1)

Chrysis splendidula Rossi, 1790 (1)

Chrysis succincta Linnaeus, 1767 (6)

Chrysura dichroa (Dahlbom, 1854) (8)

Chrysura radians (Harris, 1776) (4)

Chrysura varicornis (Spinola, 1838) (2)

Cleptes afer Lucas, 1849 (1)

Cleptes chevrieri Frey-Gessner, 1887 (6)

Cleptes nitidulus (Fabricius, 1793) (4)

Cleptes semiauratus (Linnaeus, 1761) (27)

Hedychridium incrassatum (Dahlbom,1854) (3)

Hedychridium mediocrum Linsenmaier,1987 (2) 
Hedychridium reticulatum Abeille, 1878 (3) Hedychridium roseum (Rossi, 1790) (18) Hedychridium scutellare (Tournier, 1878) (14) Hedychrum gerstaeckeri (Chevrier, 1869) (10) Hedychrum micans Lucas, 1849 (2) Hedychrum nobile (Scopoli, 1763) (20) Holopyga amoenula Dahlbom, 1845 (2) Holopyga chrysonotus (Förster, 1853) (7) Holopyga ignicollis Dahlbom, 1854 (2) Omalus auratus (Limnaeus, 1758) (10) Omalus biaccinctus (Buysson, 1892) (1) Omalus bidentulus (Lepeletier, 1806) (2) Omalus punctulatus Dahlbom, 1854 (2) Omalus violaceus (Scopoli, 1763) (1) Philoctetes micans (Klug, 1835) (1) Trichrysis cyanea (Linnaeus, 1758) (19)

\section{CIMBICIDAE}

Abia sericea (Linnaeus, 1767) (1) Corynis ? andrei (Konow, 1898) (1) Corynis italica (Lepeletier, 1823) (1) Corynis obscura (Fabricius, 1775) (7)

\section{CYNIPIDAE}

Andricus curvator Hartig, 1840 (4) Andricus foecundatrix (Hartig, 1840) (1) Andricus kollari (Hartig, 1843) (*) Andricus nudus Adler, 1881 (2) Andricus pictus (Hartig, 1856) (*) Andricus quercusradicis (Fabricius, 1798) (2) Andricus quercustozae (Bosc, 1792) (*) Andricus testaceipes (Hartig, 1843) (1) Andricus solitarius (B. de Fonsc., 1832) (6) Andricus sp 1 (1)

Andricus sp 2 (2)

Aulacidea hieracii (Bouché, 1834) (1) Aylax papaveris (Perris, 1839) (3) Cecconia valerianellae (Thomson, 1877) (1) Ceroptres clavicornis Hartig, 1840 (1) Diplolepis mayri (Schlechtendal, 1877) (2) Diplolepis nervosa (Curtis, 1838) (5) Diplolepis rosae (Linnaeus, 1758) (*) Isocolus scabiosae (Giraud, 1859) (1) Liposthenes kerneri (Wachtl, 1891) (3) Neuroterus quercusbaccarum (L., 1758) (*) Neuroterus tricolor (Hartig, 1841) (1) Periclistus brandtii (Ratzeburg, 1832) (3) Periclistus caninae Hartig, 1840 (18) Phanacis hypochoeridis (Kieffer, 1887) (1) Phanacis sp (1)

Plagiotrochus coriaceus (Mayr, 1882) (3) Plagiotrochus quercusilicis (Fabricius, 1798) (3) Saphonecrus connatus (Hartig, 1840) (59) Synergus albipes Hartig, 1841 (2) Synergus apicalis Hartig, 1841 (4) Synergus crassicornis (Curtis, 1838) (1) Synergus gallaepomiformis (B. de Fonsc., 1832) (12) Synergus ilicinus (Barbotin, 1972) (1) Synergus incrassatus Hartig, 1840 (2) Synergus pallidipennis Mayr, 1872 (1) Synergus $s p$ (1)

Synergus umbraculus Olivier,1791 (3) Timaspis lampsanae Perris, 1873 (4) Trigonaspis synaspis (Hartig, 1841) (2) Xestophanes potentillae (Retzius in D.,1873) (1)

\section{DRYINIDAE}

Anteon jurineanum Latreille, 1809 (4) Anteon arcuatum Kieffer, 1905 (1) Anteon ephippiger (Dalman, 1818) (2) Anteon flavicorne (Dalman, 1818) (5) Anteon fulviventre (Haliday, 1828) (33) Anteon gaullei Kieffer, 1905 (4) Anteon pubicorne (Dalman, 1818) (76) Anteon tripartitum Kieffer, 1905 (6) Aphelopus atratus (Dalman, 1823) (56) Aphelopus camus Richards, 1939 (8) Aphelopus melaleucus (Dalman, 1818) (27) Aphelopus serratus Richards, 1939 (11)
Echthrodelphax hortusensis (Abdul-Nour) (1) Gonatopus albosignatus Kieffer, 1905 (13) Gonatopus distinctus Kieffer (5)

Gonatopus helleni (Rootikainen, 1961) (42) Gonatopus nearcticus (Fenton, 1927) (1) Gonatopus sepsoides Westwood, 1833 (29) Gonatopus striatus Kieffer (1)

Lonchodryinus ruficornis (Dalman, 1818) (98) Gonatopodinae (16)

\section{ELASMIDAE}

Elasmus sp 1 (1)

Elasmus sp 2 (3)

Elasmus sp 3 (23)

Elasmus sp 4 (1)

\section{EMBOLEMIDAE}

Embolemus rudii Westwood, 1833 (1)

\section{ENCYRTIDAE}

Caenocercus puncticollis (Thomson, 1876) (1)

Copidosoma agrotis (Fonscolombe, 1832) (3)

Copidosoma hispanicum (Mercet, 1921) (1)

Copidosoma truncatellum (Dalman, 1820) (5) Cowperia areolata (Walker, 1872) (1) Homalotyloidea dahlbomi (Westwood, 1837) (7) Mahencyrtus comara (Walker, 1837) (2) Metaphycus insidiosus (Mercet, 1921) (7) Metaphycus melanostomatus (Timberlake, 1916) (2) Metaphycus parvus (Mercet, 1921) (7) Metaphycus stagnarum Hoffer, 1954 (3) Metaphycus zebratus (Mercet, 1917) (10) Metaphycus sp (1)

Microterys sp (3)

Rhopus sp (7)

Syrphophagus aphidivorus (Mayr, 1876) (2)

Trechnites flavipes (Mercet, 1921) (1)

Tricnemus humilis Mercet (1)

Tugahania hemipennis (Ratzeburg) (5)

Encyrtidae no ident. (1195)

\section{EULOPHIDAE}

Achrysocharoides atys (Walker, 1839) (5)

Asecodes coronis (Walker, 1838) (5)

Asecodes lagus (Walker, 1838) (1)

Ceranisus menes (Walker, 1839) (4)

Chrysocharis clinius (Walker, 1839) (1) Chrysocharis entedonoides (Walker, 1872) (1)

Chrysocharis gemma (Walker, 1839) (45)

Chrysocharis nephereus (Walker, 1839) (1)

Chrysocharis pilosa Delucchi, 1954 (2)

Chrysocharis polyzo (Walker, 1839) (3)

Chrysocharis prodice (Walker, 1839) (5)

Chrysocharis pubicornis (Zetterstedt, 1838) (1)

Chrysocharis $s p$ (1)

Chrysocharis submutica Graham, 1963 (15)

Chrysocharis viridis (Nees, 1834) (1)

Chrysonotomyia sp. nr germanica Erdos, 1956 (7)

Chrysonotomyia sp. nr lyonetiae (Ferriere, 1952) (1)

Chrysonotomyia chlorogaster (Erdös, 1966) (6)

Chrysonotomyia ruforum (Krausse, 1917) (1)

Cirrospilus curvineurus Askew, 1965 (4)

Cirrospilus lyncus Walker, 1838 (15)

Cirrospilus pictus (Nees, 1834) (1)

Cirrospilus sp. nr vittatus Walker, 1838 (1)

Cirrospilus staryi Boucek, 1959 (1)

Cirrospilus viticola (Rondani, 1877) (1)

Cirrospilus vittatus Walker, 1838 (67)

Dermatopelte budensis Erdös \& Novicky, 1951 (1)

Diaulinopsis arenaria (Erdös, 1951) (75) Dicladocerus westwoodii Westwood, 1832 (4)

Diglyphus chabrias (Walker, 1838) (11)

Diglyphus crassinervis Erdös, 1958 (2)

Diglyphus isaea (Walker, 1838) (569)

Diglyphus poppoea Walker, 1848 (26) Diglyphus sp (1)

Elachertus sp. nr bisurmanus Erdös, 1966 (1)

Elachertus (Hyssopus) nigritulus (Zetterstedt,1838) (3)

Entedon crassiscapus Erdös, 1944 (3)

Entedon diotimus Walker, 1839 (1)
Entedon ergias Walker, 1839 (12)

Entedon fufius Walker, 1846 (1)

Entedon gracilior Graham, 1971 (11)

Entedon hercyna Walker, 1839 (4)

Entedon molibdaenus Erdös, 1944 (8)

Entedon rumicis Graham, 1971 (5)

Entedon sp (1)

Entedon sp nr fufius Walker, 1846 (1)

Entedon sp nr rumicis (2)

Entedon sp nr urticarii Erdös, 1951 (1)

Euderus albitarsis (Zetterstedt, 1838) (24)

Euderus sp (1)

Eulophus ramicornis (Fabricius, 1781) (1)

Euplectrus bicolor (Swederus, 1795) (18)

Goetheana shakespearei Girault

Hemiptarsenus dropion (Walker, 1839) (39)

Hemiptarsenus fulvicollis Westwood, 1833 (1)

Hemiptarsenus unguicellus (Zetterstedt, 1838) (38)

Ionympha sp (1)

Microlycus virens Erdös, 1951 (9)

Necremnus artynes (Walker, 1839) (1)

Necremnus cosconius (Walker, 1839) (37)

Necremnus folia (Walker, 1839) (7)

Necremnus leucarthros (Nees, 1834) (88)

Necremnus metalarus (Walker, 1839) (9)

Necremnus tidius (Walker, 1839) (54)

Neochrysocharis albiscapus Erdos, 1954 (3)

Neochrysocharis aratus (Walker, 1838) (15)

Neochrysocharis arvensis Graham, 1963 (13)

Neochrysocharis chlorogaster (Erdös, 1966) (17)

Neochrysocharis clinias (Walker, 18398 (2)

Neochrysocharis cuprifrons Erdös, 1954 (1)

Neochrysocharis formosa (Westwood, 1833) (78)

Neochrysocharis sp 1 (1)

Neochrysocharis sp 2 (1)

Neochrysocharis sp 3 (1)

Omphale acamas (Walker, 1839) (16)

Omphale aetius (Walker, 1839) (1)

Omphale lugens (Nees, 1834) (3)

Omphale telephe (Walker, 1839) (1)

Omphale versicolor (Nees, 1834) (4)

Parasecodella obscura (Thomson, 1878) (1)

Pediobius acantha (Walker, 1839) (14)

Pediobius brachycerus (Thomson, 1878) (3)

Pediobius epigonus (walker, 1839) (1)

Pediobius eubius (Walker, 1839) (6)

Pediobius phragmitis Boucek, 1965 (1)

Pediobius pyrgo (Walker, 1839) (1)

Pediobius sp (1)

Pnigalio longulus (Zetterstedt, 1838) (1)

Pnigalio pectinicornis (Linnaeus, 1758) (39)

Pnigalio soemius (Walker, 1839) (159)

Ratzeburgiola cristata (Ratzeburg, 1848) (43)

Rhicnopelte crassicornis (Nees, 1834) (1)

Stenomesius rufescens (Rossius, 1790) (5)

Sympiesis acalle (Walker, 1848) (9)

Sympiesis asecta Delucchi, 1962 (2)

Sympiesis euspilapterygis (Erdös, 1958) (1)

Sympiesis flavopicta Boucek, 1959 (3)

Sympiesis gregori Boucek, 1959 (53)

Sympiesis sericeicornis (Nees, 1834) (4)

Teleopterus erxias (Walker, 1848) (2)

Xanthellum transsylvanicum Erdös, 1951 (6)

Tetrastichinae (3487)

EUPELMIDAE

Anastatus bifasciatus (Geoffroy, 1785) (6)

Anastatus giraudi (Ruschska, 1921) (1)

Calosota aestivalis Curtis, 1836 (4)

Calosota vernalis Curtis, 1836 (21)

Calymmochilus subnubilus (Walker, 1872) (2)

Eupelmus (Macroneura) vesicularis (Retzius, 1783) (1)

Eupelmus sp. (109)

Eusandalum inerme (Ratzeburg, 1848) (1)

Metapelma nobilis (Förster, 1860) (1)

Reikosiella (Hirticauda) hungarica Erdös (1)

EVANIIDAE

Brachygaster minutus (Olivier, 1791) (356) 


\section{FIGITIDAE}

\section{Anacharitinae}

Anacharis immunis Walker, 1835 (6)

Anacharis eucharioides (Dalman, 1818) (4)

Xyalaspis armata (Giraud, 1860) (1)

Xyalaspis $s p 1$ (1)

Xyalaspis sp 2 (13)

Xyalaspis sp 3 (71)

Figitinae

Figites sp 1 (1)

Figites $s p 2$ (1)

Lonchidia $s p(2)$

Melanips opacus (Hartig, 1840) (1)

Melanips alienus Giraud, 1860 (6)

Aspicerinae

Aspicera sp (1)

Callaspidia $s p(45)$

Charipinae

Apocharips xantocephala (Thomson, 1862) (6)

Alloxysta sp 1 (34)

Alloxysta sp 2 (2)

Alloxysta sp 3 (1)

Alloxysta sp 4 (5)

Alloxysta sp 5 (4)

Alloxysta sp 6 (1)

Alloxysta sp 7 (2)

Alloxysta sp 8 (1)

Alloxysta sp 9 (1)

Alloxysta spp (248)

Dilyta subclavata Foerster, 1869 (5)

Phaenoglyphis sp 1 (1)

Phaenoglyphis sp 2 (9)

Phaenoglyphis sp 3 (2)

Phaenoglyphis sp 4 (1)

\section{Eucoilinae}

Cothonaspis gracilis Hartig, 1841 (11)

Cothonaspis longula Nordlander, 1976 (20)

Cothonaspis pentatoma Hartig, 1840 (2)

Diglyphosema eupatorii Foerster, 1869 (8)

Disorygma depile (Giraud, 1860) (1)

Ganaspis mundata Foerster, 1869 (3)

Glauraspidia copulata (Foerster, 1869) (3)

Glauraspidia microptera (Hartig, 1840) (1)

Gronotoma allotriaeformis (Giraud, 1860) (11)

Hexacola hexatoma (Hartig, 1841) (7)

Kleidotoma caledonica Cameron, 1888 (31)

Kleidotoma filicornis Cameron, 1889 (7)

Kleidotoma gracilicornis Cameron, 1889 (9)

Kleidotoma sp 1 (4)

Kleidotoma sp 2 (1)

Kleidotoma nigra (Hartig, 1840) (17)

Kleidotoma picipes Cameron, 1886 (1)

Kleidotoma psiloides Westwood, 1833 (11)

Kleidotoma striata Cameron, 1886 (14)

Kleidotoma striaticollis Cameron, 1880 (29)

Kleidotoma tetratoma Thomson, 1861 (17)

Kleidotoma tomentosa (Giraud, 1860) (1)

Kleidotoma truncata Cameron, 1889 (10)

Rhoptromeris heptoma(Hartig, 1840) (18)

Rhoptromeris villosa (Hartig, 1841) (75)

Trybliographa sp 1 (1)

Trybliographa sp 2 (1)

Trybliographa sp 3 (1)

Trybliographa trichopsila (Hartig, 1841) (1)

\section{FORMICIDAE}

Anergates atratulus (Schenck, 1852) (6)

Aphaenogaster gibbosa (Latreille, 1798) (2)

Aphaenogaster iberica Emery, 1908 (5)

Aphaenogaster senilis Mayr, 1853 (17)

Camponotus foreli Emery, 1881 (1)

Camponotus piceus (Leach, 1825) (2)

Camponotus sp (19)

Cataglyphis iberica (Emery, 1906) (1)

Crematogaster auberti Emery, 1869 (7)

Diplorhoptrum $s p(13)$

Formica pratensis Retzius, 1783 (24)

Formica sanguinea Latreille, 1798 (10)
Formica sp (1)

Hypoponera eduardi (Forel, 1894) (33)

Lasius sp 1 (23)

Lasius sp 2 (68)

Leptanilla $s p(1)$

Leptothorax sp 1 (29)

Leptothorax sp 2 (1)

Messor sp (2)

Myrmica aloba Forel, 1909 (1)

Myrmica sp (55)

Myrmicinae (1)

Plagiolepis pygmaea (Latreille, 1798) (3)

Stenamma sp (2)

Tapinoma erraticum (Latreille, 1798) (120)

Tapinoma nigerrimum (Nylander, 1886) (116)

Tetramonium caespitum (Linnaeus, 1758) (5)

\section{HELORIDAE}

Helorus sp. (15)

\section{ICHNEUMONIDAE}

Absyrtus veridicator Aubert, 1979 (6)

Acrotomus succintus (Gravenhorst) (7)

Adelognathus chrysopygus (Gravenhorst, 1829) (1)

Adelognathus dorsalis (Gravenhorst, 1829) (1)

Adelognathus nigriceps Thomson, 1888 (1)

Adelognathus pallipes (Gravenhorst, 1829) (1)

Adelognathus punctulatus (Thomson, 1883 (1)

Adelognathus tenthredinarum (Giraud, 1872) (2)

Aethecerus graniger Thomson (1)

Alomya sp. (2)

Alloplasta piceator (Thunberg, 1822) (4)

Aneuclis incidens (Thomson, 1889) (130)

Aneuclis melanarius Holmgren, 1860 (9)

Banchus crefeldensis Ulbricht, 1916 (11)

Banchus moppiti Fitton, 1985 (4)

Banchus pictus Fabricius, 1798 (1)

Barycnemis harpura (Schrank, 1802) (1)

Barytarbes sp. (1)

Batakomacrus crassicaudatus Kolarov, 1986 (3)

Centererus major Wesmael (1)

Centeterus sp (1)

Chorinaeus brevicalar Thomson,1887 (7)

Chorinaeus talpa (Haliday,1839) (2)

Clistopyga incitator (Fabricius, 1793) (15)

Colpognathus celerator (Gravenhorst) (16)

Collyria calcitrator (Gravenhorst, 1807) (1)

Colpotrichia elegantula (Schrank, 1781) (2)

Cremastus brevigenalis Kolarov, 1996

Cryptinae (3281)

Cryptopimpla calceolata (Gravenhorst, 1829) (71)

Cycosis rubiginosa (Gravenhorst) (3)

Diadromus collaris (Gravenhorst) (1)

Diaparsis frontalis Holmgren (1)

Diaparsis frontella Holmgren, 1860 (2)

Dicaelotus ruficoxatus (Gravenhorst) (6)

Dicaleotus sp. (2)

Diplazon laeatorius (Fabricius, 1781) (75)

Diplazon pectoratorius (Thunberg, 1822) (5)

Diplazon tetragonus (Thunberg,1822) (7)

Diplazon tibiatorius (Thunberg, 1822) (8)

Dolichomitus agnoscendus (Roman, 1939) (2)

Dolichomitus imperator (Kriechbaumer, 1854)

Dolichomitus messor (Gravenhorst, 1829) (1)

Dolichomitus subglabratus (Perkins, 1943) (2)

Dusona aversa (Foerster) (2)

Dusona erythrogaster (Foerster) (4)

Dusona humilis (Foerster) (3)

Dusona inermis (Foerster) (2)

Dusona obliterata (Holmgren) (1)

Dusona sp. nov (40)

Dusona stragifex (Foerster) (4)

Endromopoda detrita (Holmgren, 1860) (20)

Enicospilus ramidulus (Linnaeus, 1758) (1)

Enizemum ornatum (Grav.,1829) (8)

Enizemum schwarzi Diller,1987 (1)

Ephialtes manifestator (Linnaeus, 1758) (3)

Erigorgus fibulator (Gravenhorst, 1829) (2)

Exeristes roborator (Fabricius, 1793) (3)
Exetastes (Leptobatus) ziegleri (Gravenhorst, 1829) (1)

Exetastes adpressorius (Thunberg, 1822) (46)

Exetastes calobatus Gravenhorst, 1829 (7)

Exochus albicinctus Holmgren,1873 (3)

Exochus australis Thomson,1895 (1)

Exochus flavomarginatus Holmgren,1856 (2)

Exochus tibialis Holmgren,1857 (14)

Gelanes simillimus Horstmann, 1981 (26)

Glypta (C.) elongata Holmgren, 1860 (3)

Glypta (G.) bifoveolata Gravenhorst, 1829 (200)

Glypta microcera Thomson, 1889 (1)

Glypta vulnerator Gravenhorst, 1829 (1)

Hadrodactylus tarsator Thomson, 1883 (1)

Heterischnus thoracicus (Gravenhorst) (2)

Heterischnus truncator (Fabricius) (1)

Heterocola similis Horstmann, 1971 (16)

Hypsicera curvator (Fabricius, 1793) (1)

Itoplectis alternans (Gravenhorst, 1829) (3)

Itoplectis insignis (Perkins, 1957) (4)

Itoplectis maculator (Fabricius, 1775) (33)

Itoplectis tunetana (Schmiedeknecht, 1914) (1)

Itoplectis viduata (Gravenhorst, 1829) (1)

Kristotomus laetus (Gravenhorst) (13)

Lagarotis debitor (Thunbers, 1822) (1)

Lamachus virgultorum (Gravenhorst, 1829.) (1)

Linycus exhortator (Fabricius) (1)

Liotryphon punctulatus (Ratzeburg, 1848) (4)

Liotryphon $s p$ (1)

Lissonota (Loxonota) ibericator Aubert, 1972 (32)

Lissonota (Loxonota) insignita Gravenhorst, 1829 (13)

Lissonota bicincta corsicator Aubert, 1972 (4)

Lissonota biguttata Holmgren, 1860 (2)

Lissonota bivittata gallicata Aubert (5)

Lissonota bivittata Gravenhorst, 1829 (2)

Lissonota buccator (Thunberg, 1822) (1)

Lissonota clypeator (Gravenhorst, 1829) (23)

Lissonota dusmeti (Seyrig, 1927) (21)

Lissonota elector Gravenhorst, 1829 (66)

Lissonota folii Thomson, 1877 (3)

Lissonota fundator (Thunberg,1822) (1)

Lissonota humerella (Thomson, 1877) (2)

Lissonota impressor Gravenhorst, 1829 (51)

Lissonota mutator Aubert, 1969 (1)

Lissonota pictocoxis Schmiedeknecht, 1900 (1)

Lissonota proxima Fonscolombe, 1854 (62)

Lissonota saturator (Thunberg, 1822) (2)

Lissonota sp. (2)

Lissonota variabilis Holmgren, 1860 (40)

Lissonota versicolor Holmgren, 1860 (2)

Mesochorinae (77)

Mesoleius sp (6)

Mesoleptidea cingulata (Gravenhorst, 1829) (1)

Microleptinae (119)

Misetus oculatus Wesmael (1)

Neleges fraditor (Gravenhorst) (7)

Neorhacodes enslini (Ruschka, 1922) (2)

Odontocolon spinipes (Gravenhorst, 1829) (1)

Odontocolon thomsoni (Clement, 1938) (4)

Ophion mocsaryi Brauns, 1889 (3)

Ophion obscuratus Fabricius, 1798 (71)

Ophion ventricosus Gravenhorst, 1829 (1)

Orthocentrus ? patulus Holmgren, 1858 (1)

Orthocentrus asper (Gravenhorst, 1829) (27)

Orthocentrus castellanus Ceballos, 1963 (5)

Orthocentrus frontator (Zetterstedt, 1838) (5)

Orthocentrus orbitator Aubert, 1963 (70)

Orthocentrus petiolaris Thomson, 1897 (3)

Orthocentrus protervus Holmgren, 1858 (6)

Orthocentrus sannio Holmgren, 1858 (8)

Orthocentrus spurius Gravenhorst, 1829 (1)

Orthocentrus stigmaticus Holmgren, 1858 (2)

Orthocentrus strigatus Holmgren, 1858 (4)

Orthopelma brevicorne Morley, 1907 (19)

Orthopelma mediator (Thunberg, 1822) (11)

Otlophorus sp (3)

Perilissus ? compressus Thomson, 1883 (1)

Perilissus? thuringiacus Schmiedeknecht, 1912 (10) 
Perilissus buccinator Holmgrren, 1857 (1) Perilissus filicornis (Gravenhorst, 1820) (3) Perilissus rufoniger (Gravenhorst, 1820) (24) Perilissus sp (8)

Perithous divinator (Rossius, 1790) (7)

Perithous scurra (Panzer, 1804) (1)

Phaeogenes sp. (6)

Phobetes leptocerus (Gravenhorst, 1820) (1)

Phradis brevicornis Horstmann, 1971 (2)

Picrostigeus sp (29)

Pimpla contemplator (Müller, 1776) (28)

Pimpla hypochondriaca (Retzius, 1783) (73)

Pimpla nigricoxa Oehlke, 1967 (10)

Pimpla spuria Gravenhorst, 1829 (114)

Pimpla turionellae (Linnaeus, 1758) (14)

Poemenia notata Holmgren, 1859 (2)

Polysphincta tuberosa Gravenhors, 1829 (1)

Probles? extensor Aubert, 1971

Probles erythrostomus Gravenhorst, 1829 (4)

Probles extensor Aubert, 1971 (6)

Probles lucidus Szepligeti, 1899 (6)

Probles neoversatus Horstmann, 1967 (1)

Probles sp (1)

Probles versatus Holmgren, 1860 (4)

Promethes sulcator (Gravenhorst,1829) (2)

Pseudopimpla pygidiator Seyrig, 1927 (1)

Pygmaeolus nitidus (Bridgman, 1889) (42)

Rhorus longicornis (Holmgren, 1860) (1)

Sathropterus pumilus Holmgren, 1860) (4)

Scambus brevicornis (Gravenhorst, 1897) (7)

Scambus calobatus (Gravenhorst, 1897) (1)

Scambus elegans (Woldstedt, 1876) (1)

Scambus nigricans (Thomsan, 1877) (19)

Schizopyga frigida Cresson, 1870

Sinarachna anomala (Holmgren, 1860)

Stenomacrus? femoralis (Holmgren, 1858) (1)

Stenomacrus? laricis (Haliday, 1839) (5)

Stenomacrus caudatus (Holmgren, 1858) (1)

Stenomacrus celer (Holmgren, 1858) (1)

Stenomacrus curvulus Thomson, 1897 10)

Stenomacrus deletus Thomson, 1897 (1)

Stenomacrus exserens Thomson, 1897 (24)

Stenomacrus merula (Gravenhorst, 1829) (10)

Stenomacrus vafer (Holmgren, 1858) (3)

Sussaba flavipes (Lucas, 1849) (13)

Synodites sp (12)

Syrphoctonus crassicus (Thomson, 1890)

Syrphoctonus elegans (Gravenhorst, 1829) (22)

Syrphoctonus nigritarsus (Gravenhorst,1829) (42)

Syrphoctonus nudus (Dasch, 1964) (1)

Syrphoctonus pictus (Gravenhorst, 1829) (1)

Syrphoctonus tarsatorius (Panzer,1809) (1)

Syrphophilus bizonarius (Gravenhorst,1829) (73)

Syzeuctus maculatorius (Fabricius, 1787)

Temelucha genalis (Szepligeti, 1899) (60)

Tersilochus? filicornis Thomson, 1889 (1)

Tersilochus caudatus Holmgren, 1858 (6)

Tersilochus jocator Holmgren, 1858 (12)

Tersilochus obscurator Aubert, 1959 (5)

Tersilochus sp 1 (1)

Tersilochus sp 2 (4)

Tersilochus sp 3 (2)

Tersilochus varius Horstmann, 1981 (4)

Triclistus lativentris Thomson,1887 (1)

Triclistus podagricus (Gravenhorst,1829) (1)

Trieces niger (Bridgman,1883) (1)

Trieces thuringiacus (Schmiedeknecht,1925) (4)

Trieces tricarinatus (Holmgren,1856) (1)

Tromatobia oculatoria (Fabricius, 1798) (7)

Tromatobia ovivora (Boheman, 1821) (9)

Tryphon atriceps Steph (21)

Tryphon nigripes Holmgren (1)

Tycherus meridionator (Aubert) (2)

Tycherus sp. (2)

Tymmophorus obscuripes (Holmgren, 1856)

Woldstedtius abdominator (Bridgman,1886) (3)

Woldstedtius biguttatus (Gravenhors, 1829) (1)
Woldstedtius citropectoralis (Schmid.,1926) Xorides fuligator (Thumberg, 1822) (2) Zaglyptus multicolor (Gravenhorst, 1829) (1) Zaglyptus varipes (Gravenhorst, 1829) (35) Zatypota bohemani (Holmgren, 1860) (1)

Zatypota percontatoria (Müller, 1776) (2)

\section{LEUCOSPIDAE}

Leucospis dorsigera Fabricius, 1775 (10)

Leucospis gigas Fabricius, 1793 (2)

\section{MEGALODONTIDAE}

Megalodontes bucephalus (Klug, 1824) (1)

Megalodontes capitalatus Konow, 1904 (1)

\section{ORMYRIDAE}

Ormyrus diffinis (Fonscolombe, 1832) (5)

Ormyrus nitidulus (Fabricius, 1804) (2)

Ormyrus orientalis Walker, 1871 (14)

Ormyrus pomaceus (Geoffroy, 1788) (18)

ORUSSIDAE

Orussus abietinus (Scopoli, 1763) (1)

PLATYGASTRIDAE

Allotropa mecrida (Walker, 1835) (12)

Amblyaspis abas (Walker, 1835) (6)

Amblyaspis cf. angustula (Thomson, 1859) (5)

Amblyaspis dolichosoma Buhl, 2001 (7)

Amblyaspis prorsa (Walker, 1835) (44)

Amblyaspis roboris (Walker, 1835) (7)

Fidiobia rugosifrons Crawford, 1916 (13)

Inostemma boscii (Jurine, 1807) (3)

Inostemma contariniae Szelényi, 1938 (3)

Inostemma foersteri Kieffer, 1914 (1)

Inostemma melicerta Walker, 1835 (6)

Inostemma nievesaldreyi Buhl, 2001 (3)

Inostemma reticulatum Szelényi, 1938 (183)

Isocybus dulcinea Buhl, 2001 (6)

Isocybus femoralis Kieffer, 1916 (6)

Leptacis ozines (Walker, 1835) (31)

Leptacis vlugi Buhl, 1997 (12)

Metanopedias lasiopterae (Kieffer, 1916) (20)

Piestopleura catilla (Walker, 1835) (12)

Piestopleura garridoi Buhl, 2001 (12)

Piestopleura nievesi Buhl, 2001 (1)

Platygaster cf. corni Kieffer, 1916 (3)

Platygaster cyrsilus Walker, 1835 (1)

Platygaster etsuhoae Buhl, 1998 (1)

Platygaster cf. etsuhoae Buhl, 1998 (1)

Platygaster euhemerus Walker, 1835 (15)

Platygaster gracilipes Huggert, 1975 (5)

Platygaster gyge Walker, 1835 (1)

Platygaster laricis Walker, 1835 (6)

Platygaster leptines Walker, 1835 (10)

Platygaster leptoptera Buhl, 1998 (3)

Platygaster luteocoxalis (Kozlov, 1966) (4)

Platygaster modesta Buhl, 1998 (64)

Platygaster molsensis Buhl, 1995 (2)

Platygaster cf. munita Walker, 1835 (12)

Platygaster munki Buhl, 1994 (10)

Platygaster nisus Walker, 1835 (86)

Platygaster cf. nisus Walker, 1835 (44)

Platygaster nottoni Buhl, 1995 (2)

Platygaster otanes Walker, 1835 (17)

Platygaster pedasus Walker, 1835 (2)

Platygaster pelias Walker, 1835 (273)

Platygaster pinaensis Buhl, 1998 (6)

Platygaster sagana Walker, 1835 (7)

Platygaster schlicki Buhl, 1995 (9)

Platygaster splendidula Ruthe, 1859-complex (283)

Platygaster striatithorax Buhl, 1994 (6)

Platygaster tisias Walker, 1835 (36)

Pseudaphanomerus hyalinatus Szelényi, 1941 (1)

Synopeas blascoi Buhl, 1998 (1)

Synopeas bohemani Buhl, 1998 (27)

Synopeas ciliatus Thomson, 1859 (1)

Synopeas compressiventris (Szabó, 1981) (1)

Synopeas craterus (Walker, 1835) (3)

Synopeas gallicola Kieffer, 1916 (7)
Synopeas gastralis Buhl, 2001 (1)

Shopeas inermis Thomson, 1859 (49)

Synopeas madridiana Buhl, 2001 (5)

Synopeas rhanis (Walker, 1835) (6)

Synopeas sosis (Walker, 1835) (21)

Synopeas cf. sosis (Walker, 1835) (7)

Synopeas trebius (Walker, 1835) (15)

Synopeas cf. trebius (Walker, 1835) (1)

Synopeas ventralis (Westwood, 1833) (1)

Trichacis pisis (Walker, 1835) (211)

\section{POMPILIDAE}

Agenioideus cinctellus (Spinola, 1808) (4)

Agenioideus nubecula (Costa, 1874) (3)

Agenioideus usurarius (Tourn.) (1)

Amblyellus hasdrubal (Kohl) (1)

Anospilus orbitalis luctigerus (Costa) (3)

Aporinellus moestus (Klug) (3)

Aporinellus sexmaculatus (Spinola, 1805) (1)

Aporus bicolor Spinola, 1808 (1)

Aporus unicolor Spinola, 1808 (6)

Arachnospila anceps (Wesmael, 1851) (20)

Arachnospila brevispinis Wahis (1)

Arachnospila nuda (Tourn.) (1)

Arachnospila silvana (Kohl, 1886) (6)

Arachnospila spissa (Schioedte, 1837) (1)

Auplopus carbonarius (Scopoli, 1763) (4)

Ceropales variegata (Fabricius, 1798) (1)

Cryptocheilus versicolor (Scopoli) f. nigripes Costa (5)

Dicyrtomellus argenteus Wahis (2)

Dipogon subintermedius (Magretti, 1886) (2)

Dipogon variegatus (Linnaeus, 1758) (4)

Episyron albonotatum (Vander Linden. 1827) (1)

Episyron gallicum (Tourn.) (1)

Evagetes crassicornis (Shuckard, 1837) (4)

Evagetes dubius (Vander Linden, 1827) (2)

Evagetes siculus (Lep.) (2)

Priocnemis parvula Dahlbom, 1845 (2)

Priocnemis pusilla (Schioedte, 1837) (3)

Priocnemis vulgaris (Dufour) f. ceballosi (Junco) (3)

Ablaxia megachlora (Walker,1835) (1)

Acrocormus semifasciatus Thomson, 1878 (1)

Apelioma restrictum Graham,1969 (2)

Arthrolytus discoideus (Nees,1834) (6)

Arthrolytus maculipennis (Walker,1836) (78)

Arthrolytus nanus Askew\& Nieves Aldrey, 1982 (2)

Asaphes suspensus (Nees,1834) (47)

Asaphes vulgaris Walker,1834 (100)

Caenacis lauta (Walker,1835) (1)

Callitula bicolor Spinola,1811 (124)

Catolaccus crassiceps (Masi,1911) (28)

Cea pulicharis Walker,1837 (3)

Cecidostiba adana Askew,1961 (2)

Cecidostiba atra Askew,1975 (1)

Cecidostiba ilicina Nieves \& Askew,1988(1)

Cheiropachus quadrum (Fabricius,1785) (16)

Chlorocytus deschampsiae Graham,1965 (80)

Chlorocytus diversus (Walker,1836) (17)

Chlorocytus harmolitae Boucek,1957 (1)

Chlorocytus pilosus Graham,1965 (1)

Chlorocytus pulchripes (Walker,1834) (16)

Chlorocytus sp 1 (35)

Chlorocytus sp 2 (7)

Chlorocytus sp 3 (28)

Chlorocytus sp 4 (2)

Chlorocytus sp 5 (3)

Chlorocytus sp $6(11)$

Chlorocytus sp 8 (3)

Chlorocytus sp 9 (1)

Chlorocytus sp 10 (6)

Chlorocytus sp 11 (1)

Chlorocytus sp 12 (5)

Chlorocytus sp 13 (1)

Chlorocytus sp 14 (2)

Chlorocytus sp 15 (4)

Chlorocytus sp 16 (4) 
Chlorocytus sp 17 (1)

Chlorocytus sp 18 (3)

Chlorocytus sp 19 (1)

Chlorocytus sp 20 (3)

Chlorocytus spenceri Graham,1965 (2)

Chlorocytus spicatus (Walker,1835) (49)

Cleonymus laticornis Walker,1837 (10)

Cleonymus obscurus Walker,1837 (5)

Coelopisthia extenta (Walker,1835) (17)

Colotrechnus subcoeruleus Thomson,1877 (1)

Conomorium amplum (Walker,1835) (1244)

Cyclogastrella clypealis Boucek,1965 (39)

Cyclogastrella deplanata (Nees,1834) (2)

Cyrtogaster clavicornis Walker,1833 (13)

Cyrtogaster vulgaris Walker,1833 (84)

Cyrtoptyx latipes (Rondani,1877) (13)

Cyrtoptyx robustus (Masi,1907) (3)

Dibrachoides dynastes (Förster,1841) (15)

Dibrachys cavus (Walker,1835) (30)

Dibrachys fuscicornis (Walker,1836) (14)

Dibrachys hians Boucek,1965 (4)

Dibrachys lignicola Graham,1969 81)

Dinarmus acutus Thomson,1878 (41)

Dinotiscus eupterus (Walker,1836) (5)

Dipara petiolata Walker,1833 (54)

Euneura augarus Walker,1844 (2)

Eunotus acutus Kurdjumov,1912 (9)

Eunotus areolatus (Raztzeburg,1848) (3)

Eunotus cretaceus Walker,1834 (5)

Eunotus obscurus Masi,1931 (2)

Gastrancistrus acontes Walker,1840 (1)

Gastrancistrus acutus Walker,1834 (2)

Gastrancistrus autumnalis (Walker,1834) (3)

Gastrancistrus crassus Walker,1834 (27)

Gastrancistrus glabellus (Nees,1834) (3)

Gastrancistrus laticornis Walker,1834 (1)

Gastrancistrus latifrons (Thomson,1876) (2)

Gastrancistrus oporinus Graham,1969 (1)

Gastrancistrus salicis (Nees,1834) (1)

Gastrancistrus sp 1 (2)

Gen. sp.ind.1 (2)

Gen. sp.ind.2 (4)

Gen. sp.ind.3 (1)

Gen. sp.ind.4 (1)

Gen. sp.ind.5 (1)

Gen. sp.ind.6 (1)

Gen. sp.ind.7 (1)

Gen. sp.ind.8 (1)

Gen. sp.ind.9 (1)

Habritys brevicornis (Ratzeburg,1844) (2)

Halticopera aenea (Walker,1833) (28)

Halticopera circulus (Walker,1833) (308)

Hemitrichus oxygaster Boucek,1965 (14)

Heydenia pretiosa Förster,1856 (1)

Hobbya stenonota (Ratzeburg,1848) (2)

Holcaeus compresus (Walker,1836) (1)

Holcaeus gorgasus (Walker,1839) (20)

Holcaeus varro (Walker,1839) (1)

Homoporus apharetus (Walker,1839) (19)

Homoporus arestor (Walker,1848) (1)

Homoporus chalcidiphagus (Walsh \& Riley,1869) (1)

Homoporus destructor (Say,1817) (4)

Homoporus febriculosus (Girault,1917) (7)

Homoporus sp. nr. febriculosus (3)

Homoporus fulviventris (Walker,1835) (42)

Homoporus gibbiscuta Thomson,1878 (1)

Homoporus luniger (Nees,1834) (16)

Homoporus pulchripes Erdös,1953 (1)

Homoporus semiluteus (Walker,1872) (3)

Homoporus sp (1)

Homoporus sp.nr.febriculosus (3)

Homoporus subniger (Walker,1835) (5)

Kaleva corinocera Graham,1957 (2)

Lampoterma bianellatum Graham,1969 (3)

Lampoterma viride (Thomson,1875) (2)

Lampoterma sp. nr. Bianellatum (2)

Lamprotatus (Skeloceras) mirabile (Delucchi,1955) (8)
Macroglenes chalybea (Haliday,1833) (1) Macroglenes varicornis (Haliday,1833) (20) Meraporus graminicola Walker,1834 (282) Merisus splendidus Walker,1835 (40) Mesopolobus aequus (Walker,1833) (4) Mesopolobus amaenus (Walker,1834) (13) Mesopolobus aspilus (Walker,1835) (5) Mesopolobus diffinis (Walker,1834) (42) Mesopolobus dubius (Walker,1834) (63) Mesopolobus fasciiventris Westwood,1833 (24) Mesopolobus fuscipes (Walker,1834) (2) Mesopolobus graminum (Hard,1950) (1) Mesopolobus incultus (Walker,1834) (94) Mesopolobus jucundus (Walker,1834) (3) Mesopolobus juniperinus V.Rosen,1958 (20) Mesopolobus laticornis (Walker)agg. (6) Mesopolobus lichtensteini (Mayr,1903) (34) Mesopolobus longicollis Graham,1969 (6) Mesopolobus maculicornis (Girault,1863) (1) Mesopolobus mediterraneus (Mayr,1903) (39) Mesopolobus morys (Walker,1848) (6) Mesopolobus nobilis (Walker,1834) (78) Mesopolobus prasinus (Walker,1834) (3) Mesopolobus sp 1 (1)

Mesopolobus sp 2 (1)

Mesopolobus subfumatus (Ratzeburg,1852) (1)

Mesopolobus tibialis (Westwood,1833) (33)

Mesopolobus typographi (Ruscha,1924) (13) Mesopolobus xanthocerus (Thomson,1878) (28)

Metastenus concinnus Walker,1834 (1)

Micradelus acutus Graham,1969 (6)

Miscogaster rufipes Walker,1833 (3) Muscidifurax raptor Girault\&Sanders,1910 (1)

Nephelomalus conspersus (Walker,1835)

Norbanus (Pycroscitoides) sp (15)

Norbanus obscurus (Masi,1922) (1)

Norbanus scabriculus (Nees,1834) (2)

Norbanus sp 1 (5)

Norbanus $s p 2$ (2)

Norbanus sp 3 (1)

Notanisus (Pannoniella) sexramosa (Erdos,1946) (3) Notanisus versicolor Walker,1837 (2) Notoglyptus virescens Masi,1917 (11) Ormocerus latus Walker, 1834 (4)

Pachyneuron aphidis (Bouché,1834) (49) Pachyneuron concolor (Förster,1841) (6) Pachyneuron formosum Walker,1833 (238) Pachyneuron grande Thomson,1878 (2)

Pachyneuron solitarium (Hartig,1838) (4)

Pachyneuron sp (3)

Peridesmia discus (Walker,1835) (21) Phaenocytus glechomae (Förster,1841) (1) Platygerrhus dolosus (Walker, 1836) (1) Platygerrhus maculatus Erdös,1957 (1) Plutothrix bicolorata (Spinola,1808) (13) Plutothrix cisae Hedqvist,1966 (1)

Plutothrix trifasciata (Thomson,1878) (1)

Pseudocatolaccus nitescens (Walker,1834) (39)

Psilocera confusa Graham,1992 (17)

Psilocera crassispina (Walker,1834) (19) Psychophagus omnivorus (Walker,1835) (13)

Pteromalus albipennis Walker,1835 (2)

Pteromalus altus (Walker,1837) (3)

Pteromalus bedeguaris (Thomson, 1878) (4) Pteromalus bifoveolatus Förster,1861 (9) Pteromalus brachygaster (Graham,1969) (1) Pteromalus cioni (Thomson, 1878) (1) Pteromalus cionobius (Erdös,1953) (36) Pteromalus dispar (Curtis,1827) (9) Pteromalus dolichurus (Thomson,1878) (6) Pteromalus elevatus (Walker,1834) (3) Pteromalus fasciatus (Thomson,1878) (12) Pteromalus grandis Walker,1835 (4) Pteromalus hieracii (Thomson,1878) (5) Pteromalus intermedius (Walker,1834) (77)

Pteromalus isarchus Walker,1834 (6)

Pteromalus musaeus Walker,1844 (2)
Pteromalus platyphilus (Walker,1836) (34) Pteromalus puparum (Linnaeus,1758) (25) Pteromalus semotus (Walker,1834) (66) Pteromalus sequester Walker,1835 (90) Pteromalus smaragdus (Graham,1969) (3) Pteromalus $s p \mathrm{nr}$ berylli (1)

Pteromalus $s p$ nr. cionobius (1)

Pteromalus sp nr. elevatus (3)

Pteromalus sp nr.sequester (18)

Pteromalus $s p$ nr.tripolii (3)

Pteromalus $s p 1$ (2)

Pteromalus sp 2 (3)

Pteromalus temporalis (Graham,1969) (18)

Pteromalus tripolii (Graham,1969) (5)

Pteromalus venustus Walker,1835 (5)

Pteromalus vibulennus (Walker,1839) (3)

Rhaphitelus maculatus Walker,1834 (8)

Rhicnocoelia constans (Walker,1836) (1)

Rhicnocoelia impar (Walker,1836) (4)

Rhopalicus brevicornis Thomson,1878 (1)

Rhopalicus guttatus (Ratzeburg, 1844) (2)

Sceptrothelys grandiclava (Walker,1835) (1)

Scutellista nigra Mercet,1910 (3)

Scutellista obscura (Förster,1878) (4)

Sedma dispar Boucek,1991 (1)

Seladerma diffine (Walker,1833) (6)

Seladerma geniculatum (Zetterstedt,1838) (2)

Seladerma sp. (1)

Semiotellus mundus (Walker,1834) (1)

Spalangia fuscipes Nees, 1834 (1)

Spalangia subpunctata Förster,1850 (1)

Spalangiopelta procera Graham,1966 (2)

Sphaeripalpus fuscipes (Walker,1833) (4)

Sphegigaster flavicornis (Walker,1833) (11)

Sphegigaster nigricornis (Nees,1834) (13)

Spilomalus? Sp (1)

Spilomalus dolichogaster Gijswit \& Graham,1986 (2)

Spilomalus quadrinota (Walker,1835) (7)

Spintherus dubius (Nees,1834) (119)

Stenetra hungarica (Szelenyi,1982) (1)

Stenetra ligustica Masi,1931 (10)

Stenomalina epistena (Walker,1835) (41)

Stenomalina gracilis (Walker,1834) (44)

Stenomalina illudens (Walker,1836) (2)

Stenomalina liparae (Giraud,1836) (8)

Stenomalina sp (1)

Stenoselma nigrum Delucchi, 1956 (6)

Stinoplus etearchus (Walker,1848) (1)

Stinoplus lapsanae Graham,1960 (2)

Syntomopus incisus Thomson, 1878 (7)

Systasis encyrtoides Walker,1834 (10)

Systasis parvula Thomson,1876 (4)

Systasis tenuicornis Walker,1834 (1)

Tomicobia seitneri (Ruschka,1924) (2)

Toxeuma fuscicorne Walker,1833 (1)

Trichomalopsis acuminatus (Graham,1969) (1)

Trichomalopsis albopilosus (Graham,1969) (1)

Trichomalopsis caricicola (Graham,1969) (2)

Trichomalopsis exiguus (Walker,1834) (28)

Trichomalopsis hemipterus (Walker,1836) (1

Trichomalopsis littoralis (Graham,1969) (5)

Trichomalus apertus (Walker,1834) (16)

Trichomalus bracteatus (Walker,1835) (160)

Trichomalus campestris (Walker,1834) (249)

Trichomalus conifer (Walker, 1836) (28)

Trichomalus conifer/gynetelus (3)

Trichomalus coryphe (Walker,1835) (3)

Trichomalus elongatus Delucchi\&Graham,1956 (30)

Trichomalus flagellaris Graham,1969 (2)

Trichomalus fulvipes (Walker,1836) (9)

Trichomalus gracilicornis (Zetterstedt,1838) (1)

Trichomalus gynetelus (Walker,1835) (6)

Trichomalus helvipes (Walker,1834) (45)

Trichomalus lepidus (Förster,1841) (11)

Trichomalus lucidus (Walker,1835) (15)

Trichomalus nanus (Walker,1836) (14)

Trichomalus perfectus (Walker,1835) (80) 
Trichomalus pexatus (Walker,1835) (4) Trichomalus posticus (Walker,1834) (5) Trichomalus robustus (Walker,1835) (2) Trichomalus rufinus (Walker,1835) (39) Trichomalus rugosus Delucchi \& Graham, Trichomalus spind. B Graham, 1969 (1) Trichomalus $s p 1$ (7) Trichomalus sp 2 (5) Trichomalus sp 3 (1) Trichomalus sp 4 (1) Trichomalus sp 5 (1) Trichomalus sp 6 (2) Trichomalus sp 7 (1) Trichomalus sp 8 (1) Trichomalus sp 9 (1) Trichomalus sp.nr.acuminatus (2) Trichomalus sp.nr.conifer (11) Trichomalus sp.nr.helvipes 1 (1) Trichomalus sp.nr.helvipes 2 (2) Trichomalus sp.nr.rugosus (1) Trichomalus sp.nr.tenellus (3) Trichomalus tenellus(Walker,1834) (71) SAPYGIDAE

Sapyga quinquepunctata (F.) (27) Sapyga octoguttata Dufour (2)

\section{SCELIONIDAE}

\section{Anteris (86)}

Apegus (2)

Aradophagus (1)

Gryon (125)

Idris (3)

Probaryconus (9)

Scelio (6)

Sparasion (12)

Teleas $(9)$

Telenomus (815)

Thoron (8)

Trimorus (153)

Trissolcus (147)

Xenemerus (47)

Scelionidae no ident. (7428)

SCOLIIDAE

Scolia hirta unifasciata (Cyrillo, 1877) (1)

\section{SPHECIDAE}

Ammophila campestris Latreille, 1809 (3) Ammophila heydeni Dahlbom, 1845 (2)

Ammophila hungarica (Mocsary, 1883) (1)

Ammophila sabulosa (Linnaeus, 1758) (15)

Ammoplanus sp (8)

Argogorytes fargei (Shuckard, 1837) (37)

Argogorytes hispanicus (Mercet, 1906) (2)

Astata apostata Mercet, 1910 (1)

Astata cobosi Giner Mari, 1946 (2)

Astata minor Kohl, 1885 (7)

Astata sp. affn apostata Mercet, 1910 (2)

Belomicrus steckii Kohl, 1923 (1)

Bembix tarsata Latreille, 1809 (11)

Cerceris arenaria (Linnaeus, 1758) (11)

Cerceris interrupta peninsularis Mercet, 1903 (5)

Cerceris quadricincta (Panzer, 1799) (9)

Cerceris quadrifasciata (Panzer, 1799) (1)

Cerceris rybyensis (Linnaeus, 1771) (2)

Cerceris sabulosa (Panzer, 1799) (2)

Crabro cribrarius Linnaeus, 1758 (2)

Crossocerus dimidiatus (Fabricius, 1781) (2)

Crossocerus elongatulus (Van der Linden, 1829) (175)

Crossocerus megacephalus (Rossi, 1790) (22)

Crossocerus podagricus (Van der Linden, 1829) (9)

Crossocerus quadrimaculatus (Fabricius, 1793) (266)

Crossocerus tarsatus (Shuckard, 1837) (10)

Crossocerus vagabundus (Panzer, 1798) (1)

Crossocerus varus Lepeletier \& Brullé, 1835 (1)

Dolichurus bicolor Lepeletier, 1845 (1)

Dolichurus corniculus (Spinola, 1808) (3)

Diodontus insidiosus Spooner, 1938 (31)
Diodontus luperus Shuckard, 1837 (35)

Diodontus tristis (Van der Linden, 1829) (3)

Ectemnius cavifrons (Thomson, 1870) (5)

Ectemnius cephalotes (Olivier, 1791) (7)

Ectemnius continuus (Fabricius, 1804) (5)

Ectemnius guttatus (Van der Linden, 1829) (8)

Ectemnius meridionalis (Costa, 1871) (1)

Ectemnius rubicola (Dufour, 1840) (23)

Ectemnius sexcinctus (Fabricius, 1775) (6)

Entomognathus brevis (Van der Linden, 1829)

Gorytes quinquefasciatus (Panzer, 1798) (1)

Gorytes sulcifrons (Costa, 1869) (32)

Harpactus formosus (Jurine, 1807) (1)

Harpactus laevis (Latreille, 1792) (4)

Harpactus tumidus (Panzer, 1801) (2)

Lestica clypeata (Schreber, 1795) (2)

Lestiphorus bicinctus (Rossi, 1794) (2)

Lindenius panzeri (Van der Linden, 1829) (6)

Lindenius pygmaeus (Rossi, 1794) (2)

Mellinus arvensis (Linnaeus, 1758) (5)

Miscophus bicolor Jurine, 1807 (2)

Miscophus eatoni Saunders, 1903 (2)

Nisson interruptus (Fabricius, 1798) (9)

Nisson maculosus (Gmelin, 1790) (2)

Nisson pratensis Mercet, 1909 (1)

Nitela lucens Gayubo \& Felton, 2000 (77)

Nitela spinolae Latreille, 1809 (1)

Nitela truncata Gayubo \& Felton, 2000 (8)

Oxybelus quattuordecimnotatus Jurine, 1807 (62)

Oxybelus subspinosus Klug, 1835 (5)

Oxybelus trispinosus (Fabricius, 1787) (1)

Oxybelus variegatus Wesmael, 1852 (4)

Passaloecus brevilabris Wolf, 1958 (2)

Passaloecus corniger Shuckard, 1837 (14)

Passaloecus gracilis (Curtis, 1834) (55)

Passaloecus insignis (Van der Linden, 1829) (3)

Passaloecus singularis Dahlbom, 1844 (27)

Passaloecus turionum Dahlbom, 1844 (3)

Pemphredon lethifer (Shuckard, 1837) (27)

Pemphredon lugubris (Fabricius, 1793) (17)

Pemphredon rugifer Dahlbom, 1844 (4)

Pemphredon sp. aff. rugifera (Dahlbom, 1844) (8)

Pison atrum (Spinola, 1808) (2)

Podalonia hirsuta (Scopoli, 1763) (50)

Podalonia tydei senilis (Dahlbom, 1843) (2)

Prionyx kirbii (Van der Linden, 1827) (5)

Psenulus concolor (Dahlbom, 1843) (36)

Psenulus fulvicornis (Schenck, 1857) (6)

Psenulus fuscipennis (Dalhbom, 1843) (11)

Psenulus schenckii (Tournier, 1889) (24)

Psenulus sp. aff. schenckii (Dahlbom, 1845) (4)

Rhopalum clavipes (Linnaeus, 1758) (6)

Solierella compedita (Piccioli, 1869) (15)

Sphex flavipennis Fabricius, 1793 (1)

Sphex funerarius Gussakovskij, 1934 (6)

Spilomena beata Blutgen, 1953 (32)

Spilomena troglodytes (Van der Linden,

Stigmus pendulus Panzer, 1804 (6)

Stigmus solskyi Morawitz, 1864 (1)

Stizus hispanicus Mocsáry, 1883 (1)

Tachysphex consocius Kohl, 1892 (9)

Tachysphex fulvitarsis (Costa, 1867) (2)

Tachysphex mediterraneus Kohl, 1883 (1)

Tachysphex obscuripennis (Schenck, 1857) (1)

Tachysphex panzeri (Van der Linden, 1829) (1)

Tachysphex pompiliformis (Panzer, 1804) (36)

Tachysphex psammobius (Kohl, 1880) (2)

Tachysphex tarsinus (Lepeletier, 1845) (11)

Tachysphex unicolor (Panzer, 1806) (2)

Tachytes panzeri Dufour, 1841 (1)

Tracheliodes quinquenotatus (Jurine, 1807) (5)

Trypoxylon attenuatum Smith, 1851 (116)

Trypoxylon clavicerum Lepeletier, 1825 (15)

Trypoxylon figulus (Linnaeus, 1758) (32)

Trypoxylon kolazyi Kohl, 1893 (2)

Trypoxylon minus Beaumont, 1945 (8)

\section{TENTHREDINIDAE}

Aglaostigma aucupariae (Klug, 1814) (32)

Allantus balteatus (Klug, 1814) (2)

Allantus cinctus (Linnaeus, 1758) (38)

Allantus cingulatus (Scopoli, 1763) (123)

Allantus didymus (Klug, 1814) (188)

Allantus viennensis Schrank, 1871 (9)

Amauronematus vittatus (Lepeletier, 1823) (1)

Ametastegia carpini (Hartig, 1837) (9)

Ametastegia equiseti (Fallen, 1808) (8)

Ametastegia pallipes (Spinola, 1808) (102)

Ametastegia tener Fallen, 1808 (89)

Aneugmenus coronatus (Klug, 1814) (5)

Aneugmenus fürstenbergensis (Konov, 1885) (2)

Aneugmenus padi (Linnaeus, 1761) (58)

Apethymus serotinus (Muller, 1776) (15)

Ardis brunniventris (Hartig, 1837) (28)

Athalia bicolor Lepeletier, 1823 (13)

Athalia circularis Klug, 1815 (3)

Athalia cordata Lepeletier, 1823 (438)

Athalia cornubiae Benson, 1931 (1)

Athalia liberta (Klug, 1813) (1)

Birka cinereipes (Klug, 1814) (3)

Blennocampa pusilla (Klug, 1814) (18)

Blennocampini gen. sp. (4)

Caliroa cerasi (Linnaeus, 1758) (1)

Cladius pectinicornis (Geoffroy, 1785) (542)

Dolerus aeneus Hartig, 1837 (3)

Dolerus sanguinicollis (Klug, 1814) (9)

Elinora sp (1)

Empria excisa (Thomson, 1871) (152)

Empria inmersa? (Klug, 1814) (1)

Empria klugii (Stephens, 1835) (1)

Endelommyia aethiops (Fabricius, 1781) (2)

Eutomostethus ephippium (Panzer, 1798) (4)

Euura mucronata (Hartig, 1840) (2)

Euura venusta (Zaddach, 1883) (1)

Fenella nigrita Westwood, 1840 (7)

Halidamia affinis (Fallen, 1807) (20)

Loderus vestigialis (Klug, 1814) (1)

Macrophya annulata (Geoffroy, 1785) (53)

Macrophya diversipes Schrank, 1782 (4)

Macrophya militaris Klug, 1817 (2)

Macrophya montana (Scopoli, 1763) (12)

Macrophya punctumalbum (Linnaeus, 1767) (24)

Macrophya sp (20)

Messa hortulana Leach, 1817 (1)

Metallus albipes (Cameron, 1875) (6)

Monophadnoides geniculatus (Hartig, 1837) (15)

Monophadnoides ruficruris (Brulle, 1832) (10)

Monophadnus? semicinctus Hartig (1)

Monophadnus sp (

Nematus cf. viridis (1)

Nematus hypoxanthus Foerster, 1854 (6)

Nematus lucidus (Panzer, 1801) (44)

Nematus myositidis (Fabricius, 1804) (15)

Nematus sp (1)

Nematus tibialis Newman, 1837 (9)

Nematus viridis Stephens, 1835 (15)

Paratenthredo dilleri Taeger, 1991 (11)

Paratenthredo merceti (Konow, 1905) (1)

Pareophora pruni (Linnaeus, 1758) (1)

Pontania pedunculi (Hartig, 1837) (1)

Pontania proxima (Lepeletier, 1823) (2)

Pontania sp

Priophorus morio (Lepeletier, 1823) (6)

Priophorus ulmi (Linnaeus, 1758) (8)

Pristiphora abreviata (Hartig, 1837) (1)

Pristiphora biscalis (Förster, 1854) (8)

Pristiphora coniceps Lindqvist, 1955 (1)

Pristiphora conjugata (Dahlbom, 1835) (2)

Pristiphora crassicornis (Hartig, 1837) (23)

Pristiphora monogyniae (Hartig, 1840) (1)

Pristiphora paedida (Konov, 1904) (5)

Pristiphora pallidiventris (Fallen, 1808) (2)

Pristiphora punctifrons (Thomson, 1871) (2) 
Pristiphora sp 1 (1)

Pristiphora sp 2 (5)

Pristiphora sp 3 (2)

Rhogogaster chlorosoma (Benson, 1943) (1)

Rhogogaster genistae (Benson, 1947) (21)

Rhogogaster picta (Klug, 1814) (2)

Stauronematus compressicornis (Fabricius, 1804) (26)

Strongylogaster lineata (Christ, 1791) (6)

Strongylogaster macula (Klug, 1814) (3)

Tenthredo meridiana Lepeletier, 1823 (1)

Tenthredo $s p 1$ (1)

Tenthredo sp 2 (4)

Tenthredo zona Klug, 1814 (1)

Tenthredopsis? coqueberti (Klug, 1814) (7)

Tenthredopsis? spreta (Lepeletier, 1823) (3)

Tenthredopsis excisa (Thomson, 1870) (54)

Tenthredopsis litterata (Geoffroy, 1785) (1)

Tenthredopsis nassata (Linnaeus, 1767) (30)

Tenthredopsis sp 1 (6)

Tenthredopsis sp 2 (24)

Tenthredopsis sp 3 (18)

Tenthredopsis? tesellata (Klug, 1814) (5)
Tomostethus nigritus (Fabricius, 1805) (3)

Trichiocampus viminalis (Fallen, 1808) (1)

\section{TETRACAMPIDAE}

Epiclerus panyas (Walker, 1839)

Platynocheilus cuprifrons (Nees, 1834)

\section{TIPHIIDAE}

Tiphia sp (48)

\section{TRICHOGRAMMATIDAE}

\section{Oligosita $s p$}

\section{VESPIDAE}

Eumeninae

Ancistrocerus auctus renimacula (Lepeletier 1841) (1)

Ancistrocerus clavipennis Th. (1)

Ancistrocerus longispinosus (Saussure, 1855) (9)

Ancistrocerus nigricornis (Curtis) (1)

Ancistrocerus parietum (1)

Eumenes coarctatus (Linnaeus, 1758) (1)

Eumenes papillarius (Christ) (1)

Eumenes punctaticlypeus Giordani-Soika, 1943 (1)

Eumenes subpomiformis Blüthgen, 1938 (1)

Gymnomerus laevipes (Shuckard, 1837) (2)
Microdynerus exilis (Herrich-Schaffer, 1839) (1)

Microdynerus timidus (Saussure, 1955) (1)

Paradontodinerus ephipium (Klug, 1817) (4)

Stenodynerus lacetanicus (Blüthg.) (1)

Symmorphus bifasciatus (L.) (1)

Polistinae

Polistes dominulus (Christ 1791) (42)

Polistes semenowi Morawitz 1889 (1)

Vespinae

Dolichovespula media (Retzius 1783) (1)

Dolichovespula omissa (Bischoff 1931) (1)

Dolichovespula sylvestris (Scopoli 1763) (15)

Vespula austriaca (Panzer 1799) (1)

Vespula germanica (Fabricius 1793) (50)

Vespula rufa (Linnaeus 1758) (10)

Vespula vulgaris (Linnaeus 1758) (114)

\section{XYELIDAE}

Xiela julii (Brebisson, 1818) (7)

Xiela obscura (Strobl, 1895) (57) 